\title{
1 A Clock-Driven Neural Network Critical for Arousal
}

4 Benjamin J. Bell ${ }^{1,2 *}$, Qiang Liu ${ }^{2 *}$, Dong Won $\mathrm{Kim}^{3}$, Sang Soo Lee ${ }^{2}$, Qili Liu ${ }^{2}$, Ian D.

5 Blum $^{2}$, Annette A. Wang ${ }^{2}$, Joseph L. Bedont ${ }^{3}$, Anna J. Chang ${ }^{3}$, Habon Issa ${ }^{2}$, Jeremiah Y. $^{2}$

6 Cohen ${ }^{3}$, Seth Blackshaw ${ }^{3}$, and Mark N. Wu ${ }^{2,3, \#}$

$9{ }^{1}$ McKusick-Nathans Department of Genetic Medicine, Johns Hopkins University,

10 Baltimore, MD 21287

112 Department of Neurology, Johns Hopkins University, Baltimore, MD 21205

$12{ }^{3}$ Solomon H. Snyder Department of Neuroscience, Johns Hopkins University, Baltimore,

13 MD 21205

$14 *$ These authors contributed equally.

$15{ }^{\#}$ Correspondence should be addressed to M.N.W. (marknwu@jhmi.edu)

16

17 


\section{Summary}

19 The daily cycling of sleep and arousal states is among the most prominent biological

20 rhythms under circadian control. While much is known about the core circadian clock ${ }^{1,2}$,

21 how this clock tunes sleep and arousal remains poorly understood ${ }^{3}$. In Drosophila, we

22 previously characterized WIDE AWAKE (WAKE), a clock-output molecule that

23 promotes sleep at night ${ }^{4,5}$. Here, we show that the function of WAKE in regulating

24 circadian-dependent neural excitability and arousal is conserved in mice. $\mathrm{mWake} \mathrm{W}^{+}$cells

25 are found in the suprachiasmatic nucleus $(\mathrm{SCN})$ and dorsomedial hypothalamus (DMH).

$26 m W a k e^{D M H}$ neurons drive wakefulness and exhibit rhythmic spiking, with greater firing

27 during the night vs the day. Loss of mWAKE leads to increased spiking of $m$ Wake ${ }^{+} \mathrm{SCN}$

28 and DMH neurons and prominent behavioural arousal, specifically during the night.

29 Single-cell sequencing, imaging, and patch-clamp experiments reveal that $m W a k e^{D M H}$

30 neurons constitute a glutamatergic/GABAergic population that projects widely, receives

31 neuromodulatory input, and acts on neuromodulatory neurons. Strikingly, broad

32 chemogenetic silencing of $\mathrm{mWake}^{+}$cells leads to profound loss of behavioural

33 responsiveness and low amplitude, low frequency electroencephalography waveforms.

34 These findings suggest that the genetic mechanisms regulating circadian control of sleep

35 and arousal are conserved across $>500$ million years of evolution and define a clock-

36 regulated neural network critical for arousal. 
Main

\section{The function of WAKE is conserved in mammals}

42 We previously identified the clock-output molecule WIDE AWAKE (WAKE) from a

43 forward genetic screen in Drosophila ${ }^{4}$. WAKE modulates the activity of arousal-

44 promoting clock neurons at night, in order to promote sleep onset and quality ${ }^{4,5}$. The

45 mammalian proteome contains a single ortholog, mWAKE (also named

46 ANKFN1/Nmf9), with 56\% sequence similarity and which is enriched in the core region

47 of the master circadian pacemaker suprachiasmatic nucleus (SCN) ${ }^{4,6}$ (Fig. 1a, Extended

48 Data Fig. 1a). To investigate whether the function of WAKE is conserved in mice, we

49 generated a putative null allele of $m$ Wake $\left(m W a k e^{(-)}\right)$using CRISPR/Cas9 (Fig. 1b and

50 see Methods). As expected, $m$ Wake expression, as assessed by quantitative PCR and in

51 situ hybridization (ISH), was markedly reduced in $m W a k e^{(--)}$mice, likely due to

52 nonsense-mediated decay (Fig. 1c, 1d). Given $m$ Wake expression in the SCN, we first

53 examined locomotor circadian rhythms and found that $m W_{a k e^{(-/)}}$mice exhibit a mild but

54 non-significant decrease in circadian period length (Extended Data Fig. 1b, 1c). These

55 results are similar to findings from fly wake mutants and mice bearing the Nmf9 mutation

56 (a previously identified ENU-generated allele of $m$ Wake) $)^{4,6}$.

Because we previously demonstrated that WAKE mediates circadian regulation of

58 sleep timing and quality in fruit flies ${ }^{4,5}$, we next assessed sleep in $m W a k e^{(--)}$mice via

59 electroencephalography (EEG). Under light:dark (LD) conditions, there was no

60 difference in the amount of wakefulness, non-rapid eye movement (NREM), or REM

61 sleep between $m W a k e^{(--)}$mutants and wild-type (WT) littermate controls (Extended Data

62 Fig. 1d). In constant darkness (DD), there is a modest main effect of genotype on

63 wakefulness $(P<0.05)$ and NREM sleep $(P<0.05)$, and a mild but significant decrease in

64 REM sleep in $m W a k e^{(-/)}$mutants (Fig. 1e). Although the amount of wakefulness did not

65 appreciably differ in $m$ Wake ${ }^{(-)}$mutants compared to controls, there was a change in the

66 distribution of wakefulness at night; mutants spent more daily time in prolonged wake

67 bouts, with some ( $40 \%$ ) exhibiting dramatically long ( $>6 \mathrm{hrs})$ bouts of wakefulness

68 (Extended Data Fig. 1e, 1f).

Because fly WAKE mainly functions at night and mice (as nocturnal animals) are generally awake at that time, we reasoned that arousal, and not sleep, may be primarily

71 affected in $m$ Wake mutants. Alterations in arousal level can be quantified across different 
72 parameters, including sleep/wake behaviour, locomotor activity, and responsiveness to

73 sensory stimuli ${ }^{7}$. Thus, we next examined baseline homecage locomotor activity

74 (Extended Data Fig. 2a). mWake ${ }^{(-/)}$mutants were markedly hyperactive during the

75 subjective night compared to littermate controls, although a mild but significant increase

76 in locomotor activity was also noted during the subjective day (Fig. 1f, 1g,

77 Supplementary Video 1 ). To rule out the possibility of $2^{\text {nd }}$ site mutations causing this

78 phenotype, we examined transheterozygous $m$ Wake $e^{(\mathrm{Nm} f 9 /-)}$ mutants, which also

79 demonstrated robust locomotor hyperactivity during the night, but not during the day

80 (Fig. 1f, 1g). Similar data were obtained for $m$ Wake $e^{(--)}$and $m W a k e^{(\mathrm{Nm} f 9 /-)}$ mice under LD

81 conditions (Extended Data Fig. 2b, 2c). Locomotor activity for heterozygous $m W a k e^{(+/-)}$

82 mice was not different from littermate controls, and there was a trend for it being reduced

83 at night, compared to $m W_{a k e}^{(-/)}(P=0.13)$ and $m W a k e^{(N m f 9 /-)}(P=0.06)$ (Fig. 1f, 1g). The

84 variability of the pronounced nighttime locomotor activity in $m W_{a k e^{(-/)}}$and $m W a k e^{(\mathrm{Nm} f 9 /-)}$

85 mutants was driven by intense stereotypic circling behaviour in a subset ( $30-40 \%)$ of

86 these animals (Supplementary Video 1). Related to this, mWake was previously

87 identified as $N m f 9$, and mutations in this gene were noted to cause circling behaviour,

88 which was interpreted to be due to deficits in vestibular function ${ }^{6}$. However, the intense

89 coordinated circling behaviour demonstrated by $m$ Wake mutants has also been observed

90 in hyperaroused mice ${ }^{8-10}$. In addition, in our swim tests, mWake ${ }^{(--)}$and $m$ Wake $e^{(N m f 9 /-)}$

91 mutants swam vigorously without impairment and never had to be rescued from potential

92 drowning (Supplementary Video 2), which indicates normal vestibular function and

93 contrasts with the findings of Zhang et al. (2015). Thus, our interpretation is that these

94 and other phenotypes described for $m W a k e^{(\mathrm{Nm} f 9 / \mathrm{Nm} f 9)}$ mutants stem from their

95 hyperarousal, although we cannot rule out subtle vestibular dysfunction requiring

96 specialized testing.

97 In addition to baseline locomotor activity, another measure of arousal is sensory

98 responsiveness ${ }^{7}$. To characterize this phenotype, we evaluated startle response to an

99 acoustic stimulus in $m W a k e^{(--)}$mutants during the subjective day and subjective night.

$100 m$ Wake $^{(--)}$mutants exhibited an increased startle response to $100 \mathrm{~dB}$ and $110 \mathrm{~dB}$ stimuli

101 during subjective night, but not during the subjective day (Fig. 1h, Extended Data Fig.

102 2d). To examine provoked locomotor arousal, we performed open-field tests and found

103 that $m$ Wake $e^{(--)}$mutants were hyperactive both during the day and night and, unlike 
104 controls, failed to demonstrate habituation (Extended Data Fig. 2e-h). Taken together,

105 these data suggest that mWAKE mainly acts to suppress arousal at night, but that specific

106 provoked conditions can also reveal underlying hyperarousal in mWake mutants during

107 the day.

108 In Drosophila, we previously showed that the large ventrolateral (1-LNv)

109 photorecipient clock neurons in wake mutants lose the rhythmicity of their firing at dawn

110 vs dusk. This phenotype results from an increase in spiking frequency specifically at

111 night in the mutants, which is likely due to a reduction in GABA sensitivity in these

112 clock neurons ${ }^{4}$. We thus asked whether loss of mWAKE would cause a similar

113 phenotype in $m W_{a k e}^{+}$clock neurons from the photorecipient core region of the SCN. To

114 genetically target $m W_{a k e}^{+}$neurons while simultaneously creating a mutant allele, we

115 generated transgenic mice where exon 5 was replaced with a tdTomato-P2A-Cre cassette,

116 which should produce a null allele $\left(m W a k e^{C r e}\right)$ (Fig. 1i). As predicted, this transgene

117 labels neurons in the core region of the SCN (Fig. 1j), and we further confirmed the

118 overall fidelity of the expression pattern of this transgene, by comparing it to data from

119 whole-brain RNAscope ISH labeling of $m W a k e^{11}$. We also assessed locomotor activity

120 in DD in $m W a k e^{(\mathrm{Cre} / \mathrm{Cre})}$ animals vs heterozygous controls and found that the homozygous

121 animals phenocopied the nighttime hyperactivity of $m W a k e^{(-/)}$and $m W a k e^{(\mathrm{Nm} f 9 /-)}$ mutants,

122 arguing that $m$ Wake $e^{C r e}$ is a bona fide $m$ Wake mutant allele (Extended Data Fig. $2 \mathrm{i}, 2 \mathrm{j}$ ).

123 Patch-clamp recordings revealed a loss of cycling of spiking frequency in

$124 m$ Wake $^{+} \mathrm{SCN}$ neurons, due to a selective increase in firing rate at night in $\mathrm{mWake} e^{(\mathrm{Cre} / \mathrm{Cre})}$

125 mutants (Fig. 1k, 11, Extended Data Fig. 3a, Supplementary Table 1). The effect of

126 mWAKE on neuronal excitability is likely cell-autonomous, as intrinsic excitability of

$127 m W a k e^{S C N}$ neurons in these mutants was increased during the night, but not the day

128 (Extended Data Fig. 3b, 3c). Also analogous to fly wake mutants, recordings from

$129 m$ Wake $e^{S C N}$ neurons revealed a decrease in GABA-evoked current at night in

$130 m$ Wake $e^{(\mathrm{Cre} / \mathrm{Cre})}$ mice (Fig. 1m, 1n). This phenotype was also observed in mutants lacking

131 the core clock protein Bmal1 ${ }^{12}$, suggesting that mWAKE and BMAL act in the same

132 pathway (Extended Data Fig. 3d, 3e). These findings suggest the basic function of

133 WAKE in clock neurons is shared between flies and mice. However, in contrast to fly

134 WAKE, mWAKE action in the $\mathrm{SCN}$ is not crucial for suppressing arousal. Conditional

135 knockout of mWAKE in the ventral forebrain, including the SCN, using the Six 3-Cre 
$136 \operatorname{driver}^{13}\left(\mathrm{Six}^{\left(\mathrm{Cre}^{+}\right)}>m \mathrm{Wake}^{(\text {flox/-) }}\right.$ mice) did not alter locomotor activity (Extended Data

137 Fig. 4a-e). Thus, we next sought to identify the brain region(s) where mWAKE acts to

138 regulate arousal.

mWAKE defines a circadian-dependent arousal circuit in the DMH

141 Beyond the SCN, mWAKE is expressed in a variety of regions across the brain, such as

142 other hypothalamic sub-regions (including the dorsomedial hypothalamus/DMH, Fig. 2a,

1432 b, also see Fig. 3a), areas implicated in arousal, the limbic system, sensory processing

144 nuclei, and limited regions in the cortex ${ }^{6,11}$. The DMH has previously been implicated in

145 the circadian regulation of arousal ${ }^{14-16}$, although the specific neurons involved remain

146 unknown. We thus asked whether $m W a k e^{D M H}$ neurons mediate circadian-dependent

147 arousal. Interestingly, in controls, these neurons had greater spiking frequency during the

148 night vs the day, which is antiphase to the SCN, but aligned with the active phase of the

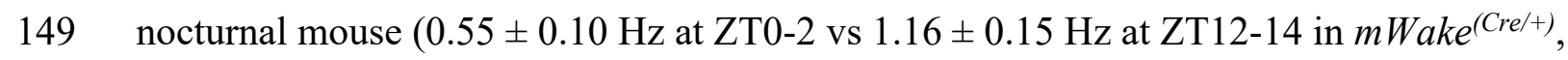

$150 P<0.01)$. Moreover, this nighttime increase was accentuated in $m W a k e^{(\mathrm{Cre} / \mathrm{Cre})}$ mutants

151 (Fig. 2c, 2d, Extended Data Fig. 5a, Supplementary Table 1). Similarly, intrinsic

152 excitability of $m W a k e^{D M H}$ neurons was greater in $m W a k e^{(\mathrm{Cre} / \mathrm{Cre})}$ mutants during the night,

153 but not the day (Extended Data Fig. 5b, 5c). These data are consistent with a circadian-

154 dependent role for $m W a k e^{D M H}$ neurons in promoting arousal.

155 To address the function of $\mathrm{mWAKE}$ in the DMH, we performed conditional

156 knockout of mWAKE. We generated a floxed allele of $m$ Wake $\left(m W a k e^{f l o x}\right)$ and

157 performed stereotaxic injection of an AAV viral vector expressing Cre-recombinase

158 (AAV-Cre) into the DMH of $m W a k e^{(f l o x f l l o x)}$ mice (Fig. 2e, 2f, Supplementary Table 2).

159 Reduction of mWAKE in the DMH led to a significant increase in locomotor activity

160 during subjective nighttime, compared to their baseline. During the subjective day, there

161 was a non-significant trend $(P=0.08)$ towards an increase in locomotor activity with

162 conditional knockout of $m$ Wake in the DMH. No differences in locomotor activity were

163 observed in sham-injected controls during the subjective day or night (Fig. 2g). These

164 findings, coupled with the observation that mWake mutants demonstrate increased

165 spiking of $m W a k e^{D M H}$ neurons at night (Fig. 2c, 2d), suggest that these neurons promote

166 arousal or wakefulness. 
To test this possibility, we chemogenetically activated these neurons by injecting an AAV vector carrying Cre-dependent DREADD-hM3Dq (AAV-DIO-DREADD-Gq) ${ }^{17}$ into the DMH of $m W a k e^{\left(\mathrm{Cre}^{++}\right)}$mice (Fig. 2h, 2i, Supplementary Table 2). CNO-mediated activation of $m W a k e^{D M H}$ neurons resulted in a substantial increase in locomotor activity, compared to vehicle-treated animals (Fig. 2j). Moreover, chemogenetic activation of $m W a k e^{D M H}$ neurons markedly increased wakefulness, with concomitant reductions in

173 NREM and REM sleep (Fig. 2k, 21, Extended Data Fig. 5d, 5e). We next performed

174 chemogenetic inhibition of $m W a k e^{D M H}$ neurons, by injecting an AAV vector encoding

175 Cre-dependent DREADD-hM4Di (AAV-DIO-DREADD-Gi) and found no significant

176 effects on locomotor activity or amount of wakefulness or NREM sleep (Extended Data

177 Fig. 5f-i, Supplementary Table 2). However, the amount of REM sleep was reduced

178 following injection of $\mathrm{CNO}$, compared to vehicle alone (Extended Data Fig. 5j). In

179 contrast, $\mathrm{CNO}$ alone administered to sham-injected $m$ Wake ${ }^{(\mathrm{Cre} /+)}$ mice did not appreciably

180 affect locomotor activity or vigilance state (Extended Data Fig. 5k, 51). Taken together,

181 these data suggest that $m W a k e^{D M H}$ neurons promote arousal and that mWAKE acts to

182 reduce arousal at night by inhibiting the activity of these neurons at that time.

183 To gain mechanistic insights into how $m W a k e^{D M H}$ neurons regulate arousal, we

184 conducted single-cell RNA sequencing (scRNA-Seq) of FACS-sorted tdTomato ${ }^{+}$cells $^{2}$

185 from the hypothalami of $m$ Wake $e^{(\text {Cre/+) }}$ mice (Fig. 3a, Extended Data Fig. 6a, 6b,

186 Supplementary Table 3). In addition to neurons, there was a significant population of

$187 \mathrm{mWake} \mathrm{H}^{+}$ependymal cells, which were likely overrepresented due to their ability to

188 survive the dissociation process (Extended Data Fig. 6a). The identity of different

189 neuronal $\mathrm{mWake}^{+}$clusters and the spatial location of these clusters were determined by

190 comparison with a hypothalamic scRNA-Seq database and using specific gene markers

191 (Extended Data Fig. 6c) ${ }^{18}$. This analysis revealed $11 \mathrm{mWake}^{+}$clusters in the

192 hypothalamus, with a prominent DMH cluster and 5 SCN-specific clusters (Fig. 3a).

193 Collectively, hypothalamic $m W_{a k e} e^{+}$neurons comprised a heterogeneous group, but were

194 largely GABAergic (most clusters including all SCN clusters) or glutamatergic (DMH

195 and VMH) (Fig. 3b). We confirmed this observation using RNAscope ISH for the SCN

196 and DMH (Extended Data Fig. 6d-g).

197 Historically, investigations of the neural basis of arousal have focused on

198 neuromodulatory circuits, but there is growing recognition that GABAergic and 
199 glutamatergic neurons likely form the core substrate for arousal, which is in turn tuned by 200 neuromodulatory networks ${ }^{3,19,20}$. We thus used our scRNA-Seq dataset to characterize 201 the repertoire of neuromodulatory receptors in $m W a k e^{D M H}$ neurons in the DMH (Fig. 3c).

$202 m W a k e^{D M H}$ neurons express specific noradrenergic, cholinergic, histaminergic, and 203 orexinergic receptors, and so we conducted rabies virus retrograde tracing (Fig. 3c, 204 Extended Data Fig. 7a, Supplementary Table 2) from these neurons to evaluate potential 205 inputs (Extended Data Fig. 7a). We observed significant retrograde labeling of histidine 206 decarboxylase ${ }^{+}$(HDC) neurons in the tuberomammillary nucleus (TMN) (Extended Data 207 Fig. 7b). We also found some labeling of orexinergic neurons in the lateral hypothalamus 208 (LH) and cholinergic neurons in the basal forebrain (BF), as well as rare labeling of 209 noradrenergic neurons in the locus coeruleus (LC) (Extended Data Fig. 7c-e).

$210 \quad$ To address whether $m W a k e^{D M H}$ neurons functionally respond to these

211 neuromodulators, we performed whole-cell patch-clamp recordings of these neurons from 212 brain slices of $m W a k e^{(\mathrm{Cre} /+)}$ mice in the presence or absence of norepinephrine, orexin, 213 acetylcholine, or histamine (Fig. 3d-f, Extended Data Fig. 7f-h, Supplementary Table 1).

214 Application of orexin and acetylcholine resulted in a significant elevation in spontaneous

215 firing rate of $m W a k e^{D M H}$ neurons in a cell-autonomous manner (i.e., in the presence of

216 synaptic blockers) (Fig. 3e, 3f). Norepinephrine increased $m W a k e^{D M H}$ neuron spiking

217 indirectly, consistent with the relative paucity of noradrenergic neurons labeled by

218 retrograde tracing from $m W a k e^{D M H}$ neurons (Extended Data Fig. 7e). In contrast,

219 administration of histamine directly reduced $m W a k e^{D M H}$ neuron firing rate (Extended

220 Data Fig. 7f-h), which may reflect a relative enrichment of the inhibitory H3 receptor 221 subtype in these neurons (Fig. 3c).

222 Neurons that comprise arousal-promoting nuclei can either act as local

223 interneurons or project broadly to influence the activity of multiple brain regions,

224 including the neocortex ${ }^{21}$. To assess which of these categories $m W a k e^{D M H}$ neurons

225 belong to, we injected an AAV vector expressing Cre-dependent eGFP (AAV-FLEX-

$226 \mathrm{eGFP}$ ) into the DMH of $m W_{a k}^{(\mathrm{Cre} /+)}$ mice and imaged the $\mathrm{GFP}^{+}$projections (Extended

227 Data Fig. 7i, 7j, Supplementary Table 2). $m W a k e^{D M H}$ neurons sent projections

228 throughout the brain, including the BF, caudate/putamen, the corpus callosum, and

229 regions in the brainstem including the LC. Prior work had suggested that the DMH

230 region may mediate circadian timing of arousal by modulating LC firing, although the 
231 responsible neurons in the $\mathrm{DMH}$ were not identified ${ }^{15}$. We therefore asked whether $232 m W a k e^{D M H}$ neurons can regulate the activity of noradrenergic LC neurons. We injected 233 an AAV vector encoding a Cre-dependent Channelrhodopsin2 (AAV-DIO-hChR2) into 234 the DMH of $m W_{a k e}^{(C r e /+)} ; T H-G F P$ mice and then performed whole-cell patch-clamp 235 recordings from noradrenergic $N E^{L C}$ neurons following blue-light stimulation of the 236 terminals of $m W a k e^{D M H}$ neurons in the LC (Fig. 3g, Supplementary Table 2). In the 237 majority of cases, optogenetic activation of $m W a k e^{D M H}$ triggered noradrenergic $N E^{L C}$ 238 excitatory post-synaptic currents (EPSCs) and spiking (Fig. 3h-j). In summary, our 239 findings suggest that glutamatergic $m W a k e^{D M H}$ neurons are arousal-promoting, project 240 widely, and bidirectionally interact with neuromodulatory networks.

The $m W_{a k} e^{+}$network is critical for arousal

243 Whether there is a "core substrate" for arousal is controversial, as silencing of various 244 genetically-defined arousal-promoting neural circuits, either alone or in combination, 245 generally leads to relatively mild phenotypes ${ }^{22-27}$. Recent models suggest that if core 246 arousal networks exist, they may be glutamatergic or GABAergic in nature, rather than

247 the classically-studied monoaminergic or cholinergic systems ${ }^{19}$. Moreover, emerging 248 data suggest that GABA or glutamate-expressing neurons located in or near previously249 defined arousal-associated nuclei are important for regulating arousal ${ }^{27-31}$. Interestingly, 250 our data suggest that $m W_{a k e}{ }^{+}$neurons are glutamatergic or GABAergic, and we have 251 recently found that these cells can be found in several regions implicated in arousal (e.g., 252 BF, TMN, vlPAG/DR, LH, PB) $)^{3,11,19,20 .}$

253 We thus hypothesized that mWAKE defines a distributed arousal network.

254 Because silencing $m W a k e^{D M H}$ neurons led to a mild phenotype (Extended Data Fig. 5h-j), 255 we chose to inhibit the broad $m W_{a k}+$ network. To do this, we crossed transgenic mice 256 expressing Cre-dependent DREADD-hM4Di $(\mathrm{LSL}-\mathrm{Gi})^{32}$ to $\mathrm{mWake} \mathrm{Cre}^{(+)}$mice to generate $257 \mathrm{mWake} \mathrm{Cre}^{(+)} ; \mathrm{LSL}-\mathrm{Gi}$ progeny. We first examined the expression pattern of the

258 DREADD-hM4Di in these mice by immunostaining and found that it largely

259 recapitulated the original expression pattern of the $m$ Wake ${ }^{(\text {Cre/+) }}$ mice (Extended Data Fig.

260 8a). We next characterized behavioural and EEG phenotypes of these mice. Within 15

261 min of CNO treatment, $m$ Wake ${ }^{\left(\mathrm{Cre}^{+}\right)} ; L S L-G i$ mice exhibited reduced spontaneous

262 locomotion and exploratory behavior. After $\sim 90 \mathrm{~min}$, we observed a profound decrease 
263 in arousal (reduced or minimal responsiveness to gentle touch or acoustic stimuli, with

264 maintenance of righting reflex) in these mice, which lasted 2-3 hrs (Fig. 4a, 4b;

265 Supplementary Video 3). Strikingly, EEG analyses of these mice revealed a marked shift

266 towards low amplitude, slow frequency waveforms following injection of CNO, but not

267 vehicle control (Fig. 4c-f; Extended Data Fig. 8b, 8c). For all but one animal, both the

268 behavioural and EEG phenotypes were reproducible and reversible, becoming

269 indistinguishable from vehicle-injected animals after $24 \mathrm{hrs}$. However, one CNO-treated

270 animal died after $>24 \mathrm{hrs}$. These phenotypes were suggestive of a stupor-like state, rather

271 than sleep, and indeed $m W a k e^{\left(\mathrm{Cre}^{+}\right)} ; L S L-G i$ mice appeared to exhibit a rebound of sleep-

272 like slow-wave activity after the effects of the CNO dissipated (Fig. 4c; Extended Data

273 Fig. 8b, 8c). For comparison, we assessed the behavioural and EEG effects of

274 chemogenetically silencing arousal-promoting $\mathrm{HDC}^{+}$(histamine decarboxylase) neurons

275 by repeating these experiments with $\mathrm{HDC}^{(\mathrm{Cre} /+)}$; LSL-Gi mice. CNO treatment of these

276 mice led to no discernable differences in behavioural responsiveness or EEG spectral

277 power and amplitude (Extended Data Fig. 8d-h). These data suggest that the $m$ Wake ${ }^{+}$

278 network is essential for basic arousal.

279 Our studies of WAKE in flies and mice suggest that the basic function of WAKE

280 is to reduce arousal at night, by inhibiting the excitability of $m W{ }^{+}$arousal-promoting

281 neurons at that time ${ }^{4,5}$. Although it is widely accepted that the circadian clock regulates

282 sleep and arousal, the cellular mechanisms remain unclear. In principle, this process

283 could be driven by direct action of SCN projections on arousal circuits ${ }^{14,15,33}$, by SCN

284 release of diffusible substances ${ }^{34-37}$, or by the activity of local clocks in arousal-

285 promoting nuclei ${ }^{38}$. Our data suggest that mWAKE mediates local clock control of

286 arousal in the $\mathrm{DMH}$, defining the first such neural circuit in this region at the genetic

287 level. Moreover, the observations that mWAKE is expressed in or near several arousal-

288 related regions ${ }^{11}$ and that $\mathrm{mWake}^{+}$neurons can project broadly (Extended Data Fig. 7j)

289 suggest a new model for clock control of arousal - multi-focal local control, which

290 allows for flexible modulatory input from environmental or internal state factors, yet also

291 facilitates coordinated time-dependent regulation throughout the brain. Although many

292 regions promoting wakefulness have been identified in the mammalian brain ${ }^{3}$, very few

293 have been shown to be critical for fundamental arousal. One prominent example is the

294 parabrachial nucleus (PB); large excitotoxic lesions in the PB lead to a coma-like state, 
295 with low amplitude, slow oscillations on EEG, a phenotype similar to that seen with

296 silencing the $m W_{a k e}^{+}$network $^{39}$. For decades, psychologists have proposed the existence

297 of distinct, but reciprocally connected, substrates for arousal: a "higher-order"

298 modulatory system and a basal network, required for attention and consciousness ${ }^{40}$. Our

299 data suggest the possibility that mWAKE marks a basal arousal network that is tuned by

300 neuromodulatory inputs and under circadian clock control.

\section{Methods}

\section{Animals}

304 All animal procedures were approved by the Johns Hopkins Institutional Animal Care

305 and Use Committee. All animals were group housed and maintained with standard chow

306 and water available ad libitum. Animals were raised in a common animal facility under a

307 14:10 hr Light:Dark (LD) cycle. Unless otherwise noted, adult male mice (2-4 months)

308 were used in all immunohistochemistry, in situ hybridization, and behavioural

309 experiments. All mouse strains were backcrossed to $C 57 B L / 6$ at least seven times prior

310 to use in behavioural experiments. Genotyping was performed either by in-house PCR

311 and restriction digest assays, or via Taq-Man based rtPCR probes (Transnetyx). The

$312 m W a k e^{N m f 9}, H D C-C r e, T H-G F P$, and Six3-Cre mice were obtained from B. Hamilton

313 (University of California, San Diego), A. Jackson (University of Connecticut), A.

314 McCallion (Johns Hopkins University), and Y. Furuta (Memorial Sloan Kettering Cancer

315 Center), respectively. Bmal1- (stock number: 009100), and LSL-Gi (stock number:

316 026219) mice were obtained from The Jackson Laboratory.

317 The $m$ Wake null mutant allele ( $m$ Wake $e^{-}$was generated by CRISPR/Cas9 genome

318 editing (Johns Hopkins Murine Mutagenesis Core), using a targeting guide RNA (gRNA:

319 CGC AGA AGA ATC CTC GCA AT) to the $4^{\text {th }}$ exon of $m$ Wake and a $136 \mathrm{bp}$

320 oligonucleotide (AGT GCG GAC TTT CTC TGG CTC CTG TCC GCA GAA GAA

321 TCC TCG GCG GAA TTC AAT GGG CAC GTT GTT GGT CAT GAT GGC GAT

322 GTC CAG GGG TGT CAG CCC TTC GCT GTT CGG TGT) containing two 64 bp

323 homology arms, surrounding an 8 bp insertion (GCGGAATT), which includes an in-

324 frame stop codon and induces downstream frameshifts. Exon 4 is predicted to be in all

$325 \mathrm{mWake}$ splice isoforms. These constructs were injected into the pronucleus of $C 57 \mathrm{BL} / 6 \mathrm{~J}$

326 fertilized zygotes and implanted into pseudopregnant females. Pups were assayed for 
327 insertion by Sanger sequencing of $m$ Wake gDNA, and knockdown confirmed via qPCR

328 and in situ hybridization. The conditional $m$ Wake knockout allele ( $m$ Wakflox $)$ was

329 generated via homologous recombination in hybrid (129/SvEv $\mathrm{C} C 57 \mathrm{BL} / 6)$ mouse

330 embryonic stem cells (Ingenious Targeting Laboratory). In the stem cells, a construct

331 containing two loxP sites and a Neomycin cassette flanking the $4^{\text {th }}$ exon of $m$ Wake was

332 integrated into the genomic DNA. These cells were then injected into $C 57 B L / 6 J$

333 blastocysts, and offspring with high agouti content were crossed to flipase (FLP)-

334 expressing $C 57 B L / 6 J$ to remove the Neomycin selective marker. Offspring were then

335 screened via Sanger sequencing to confirm proper insertion of both LoxP loci. A

336 transgenic mouse line expressing both Cre recombinase and tdTomato in $m W_{a k e}^{+}$cells

$337\left(m W a k e^{C r e}\right)$ was generated via homologous recombination in hybrid $(129 / S v E v$ x

$338 C 57 B L / 6$ ) mouse embryonic stem cells (Ingenious Targeting Laboratory). The knock-in

339 vector targeted exon 5 of the $m$ Wake locus and was integrated $21 \mathrm{bp}$ into exon 5 ,

340 replacing the remainder of the exon with a tdTomato-P2A-split Cre-Neo-WPRE-BGHpA

341 cassette, which causes frameshifted nonsense mutations downstream, resulting in an

342 mWake loss-of-function allele. Neomycin was excised via crossing to FLP mice, and

343 offspring sequenced to confirm the inclusion of the whole sequence into the $m$ Wake

344 locus.

\section{Molecular Biology}

347 To quantify $m$ Wake transcript in the $m W a k e^{(-/)}$mutant mice, qPCR was performed.

348 Hypothalami were dissected at $\sim$ ZT0, and RNA extracted using Trizol Reagent

349 (Invitrogen). qPCR was performed using a SYBR PCR master mix (Applied

350 Biosystems) and a 7900 Real Time PCR system (Applied Biosystems), with the

351 following primers, which target exon 4: mWake-F: 5'-CCC TAA CGG TCA GCT TTC

352 AAG A-3' and mWake-R: 5'-GAC ATG CTC CAT TCC ACT TTG TAC-3'. GAPDH

353 was used as an internal control. Ct value was compared against regression standard curve

354 of the same primers. 3 biological replicates were performed.

\section{Single Cell Sequencing}

357 Seven week old, male $m W_{a k} e^{(\mathrm{Cre} /+)}$ mice were processed at $\sim$ ZT5 for single-cell RNA-

358 Sequencing (scRNA-Seq). A modified Act-Seq ${ }^{41}$ method was used in conjunction with a 
359 previously described dissociation protocol $^{18}$, with supplementation of Actinomycin D

360 during dissociation $(45 \mu \mathrm{M})$ and after final resuspension $(3 \mu \mathrm{M})$, following debris

361 removal (Debris Removal Solution (130-109-398, MACS Miltenyi Biotec) in between. 1

$362 \mathrm{~mm}$ hypothalamic sections between Bregma $0.02 \mathrm{~mm}$ (collecting medial and lateral

363 preoptic area) and Bregma $-2.92 \mathrm{~mm}$ (beginning of the supramammillary nucleus) were

364 collected, and 2-3 mice pooled per scRNA-Seq library.

$365 \quad$ Following dissociation, tdTomato $^{+}$cells were flow-sorted using an Aria IIu Sorter

366 (Becton Dickinson). Between 400 - 1000 cells were flow-sorted per brain. Flow-sorted

367 cells were pelleted and re-suspended in $47.6 \mu \mathrm{l}$ resuspension media. $1 \mu \mathrm{l}$ of flow-sorted

368 tdTomato $^{+}$cells were used to quantify $\%$ of tdTomato $^{+}$cells with a phase-contrast

369 microscope. Only samples containing $\sim 99 \%$ flow-sorted tdTomato ${ }^{+}$cells were processed

370 for scRNA-Seq. The remaining $46.6 \mu \mathrm{l}$ were used for the 10x Genomics Chromium

371 Single Cell system (10x Genomics, CA, United States) using V3.0 chemistry per

372 manufacturer's instructions, generating a total of 3 libraries. Libraries were sequenced on

373 Illumina NextSeq 500 with $\sim 150$ million reads per library ( $\sim 200,000$ median reads per

374 cell). Sequenced files were processed through the CellRanger pipeline (v 3.1.0, 10x

375 Genomics) using a custom mm10 genome (with tdTomato-P2A-Cre-WPRE-bGH

376 sequence). All 3 libraries were aggregated together for downstream analysis.

377 Seurat V3 ${ }^{42}$ was used to perform downstream analysis following the standard

378 pipeline, using cells with more than 200 genes and 1000 UMI counts, removing $m$ Wake-

379 tdTomato ${ }^{+}$ependymal cells and non-mWake-cells ( $\sim 1 \%$ of the total cluster composed of

380 oligodendrocytes and astrocytes) using known markers genes in the initial clustering ${ }^{18}$.

381 Louvain algorithm was used to generate different clusters, and spatial information (spatial

382 location of different $m$ Wake-tdTomato $^{+}$clusters across hypothalamic nuclei) and identity

383 of neuronal clusters were uncovered by referring to a hypothalamus scRNA-Seq

384 database ${ }^{18}$. Region-specific transcription factors expressed in $\mathrm{mWake}^{+}$neurons were used

385 to train $m$ Wake-scRNA-Seq. Spatial information of different $m$ Wake neuronal

386 populations were further validated by matching to the Allen Brain Atlas ISH data using

387 cocoframer ${ }^{43}$, as well as matching to known $m$ Wake neuronal distribution across the

388 hypothalamus ${ }^{32}$. The percentage of GABAergic $\left(\right.$ Slc32al $\left.1^{+}\right)$and Glutamatergic

$389\left(\right.$ Slc17a6 $\left.{ }^{+}\right)$neurons within each cluster was calculated. 
Receptors for norepinephrine, dopamine, acetylcholine, histamine and orexin were identified in the scRNA-Seq dataset, and the normalized gene expression within

392 cluster $4(\mathrm{DMH})$ was calculated. scRNA-seq data from this study are accessible through

393 GEO Series accession number GSE146166.

395 Behavioural Analysis

396 Animals were entrained to a 12:12 hr LD cycle for at least 2 weeks before any locomotor 397 or EEG-based behavioural experiments.

399 Homecage locomotor activity: Animals were separated into new individual cages with

400 access to food and water ad libitum and allowed to acclimate for 4 days before data

401 collection. Data were recorded over 2 days of 12:12 hr LD and 2 days of constant

402 darkness (DD) cycles. Locomotor activity was recorded and analyzed using the Opto M3

403 monitoring system with IR beams spaced 0.5 inch apart and Oxymax data-acquisition

404 software (Columbus Instruments). Total activity (the total number of beam breaks along

405 the $\mathrm{X}$ and $\mathrm{Y}$ axis) was measured in $10 \mathrm{~s}$ intervals. Locomotor activity profiles were

406 generated from the $2^{\text {nd }}$ day of LD or the $1^{\text {st }}$ day of DD.

408 Wheel-running activity: Adult male mice (3-5 months) were placed into individual 409 cages with a vertical running wheel (ActiMetrics) and ad libitum access to food and 410 water. After 1 week of acclimatization in wheel-cages under 12:12 hr LD conditions, 2

411 weeks of LD wheel-running activity were recorded, followed by 2 weeks of free-running 412 activity in DD. Wheel-running data were acquired in $1 \mathrm{~min}$ bins and analyzed using

413 ClockLab software (ActiMetrics). Period estimates were calculated using data from 12 414 days of DD.

416 Open Field Test (OFT): OFT were conducted in $9 \times 11$ inch polyethylene cages using the 417 Opto M3 beam-break setup described above. Auto-Track software (Columbus

418 Instruments) was used to record the X-Y position, distance, and speed of each mouse at

$41910 \mathrm{~Hz}$ frequency. During the $3 \mathrm{hr}$ test, animals were in constant darkness without

420 bedding, food, or water. Each animal was placed in the arena at the indicated time point, 421 and total activity and distance traveled were summed in 5 min bins as readouts. 
422 Behavioural data were analyzed using a custom MATLAB (Mathworks) program.

423 Habituation was calculated by summing the total distance of the first $30 \mathrm{~min}$ of the trial

424 and comparing it to the total distance of the final $30 \mathrm{~min}$ of each trial.

426 Acoustic Startle: Acoustic startle response was recorded using an SR-LAB Startle

427 Response System (San Diego Instruments) apparatus, which consists of a sound-isolating

428 cabinet containing a pressure-sensitive plate. Mice were placed into a plexiglass tube

429 (I.D. $5 \mathrm{~cm}$ ) and then enclosed inside the chamber on the pressure-recording plate for the

430 duration of the trial. Mice were acclimated to the test environment, including $50 \mathrm{~dB}$ of

431 background white noise, for $5 \mathrm{~min}$ before trials began. Each trial consisted of a $20 \mathrm{~ms}$

432 white noise stimulus $(100 \mathrm{~dB}, 110 \mathrm{~dB}$, or $120 \mathrm{~dB})$ presented from a speaker $20 \mathrm{~cm}$ above

433 the mouse's head. The response of the animal in the $100 \mathrm{~ms}$ afterwards was recorded as

434 vibration intensity on the pressure platform (in millivolts, $\mathrm{mV}$ ); Vavg was the total

435 activity averaged over the recorded window, while Vmax was the peak response

436 intensity. All three trial tones were repeated 5 times throughout the experiment, in a

437 pseudorandom order and separated by pseudorandom inter-trial intervals (13-17

438 s). Trials with significant vibration $100 \mathrm{~ms}$ before the tone were excluded from the

439 analysis ( $<5$ instances).

441 Behavioural assessment of reduced arousal: $m W a k e^{\left(\text {Cre/+) }^{2}\right.} ; L S L-G i$ mice were assessed 90

$442 \mathrm{~min}$ after IP injection of vehicle or $0.3 \mathrm{mg} / \mathrm{kg}$ CNO. Behaviour was scored by a blinded

443 investigator, and each mouse classified as "Normal," "Reduced Reactivity," or

444 "Stuporous," based on the individual's spontaneous behaviour and response to gentle

445 handling. "Normal" mice spontaneously explored the environment and briskly responded

446 to stimulation. "Reduced Reactivity" mice were generally immobile, but would try to

447 evade handling. "Stuporous" mice did not exhibit spontaneous locomotion, and had a

448 minimal response to handling. All animals exhibited righting reflex.

\section{Electroencephalography (EEG)}

451 Surgery: 8-10 week old male mice were anesthetized to surgical depth with a

452 ketamine/xylazine mixture $(100 \mathrm{mg} / \mathrm{kg}$ and $10 \mathrm{mg} / \mathrm{kg}$, respectively), and all fur was

453 removed from the top of the head. A skin incision was made along the top of the skull in 
454 the rostral-caudal direction, and the scalp was cleaned and connective tissue removed.

455 The 3-channel EEG headmount (Pinnacle Technology) was aligned with the front $3 \mathrm{~mm}$

456 anterior to the bregma and glued to the top of the skull. Four guideholes were hand-

457 drilled, and screws inserted to attach the headmount. EMG wires were then inserted into

458 the left and right neck muscles. After skin closing, the headmount was sealed to the skull

459 using dental cement. All animals recovered from surgery for $>5$ days before being

460 affixed to the EEG recording rigs.

462 EEG Recording: Sleep behavioural data were obtained using the Pinnacle Technology

463 EEG/EMG tethered recording system. Following recovery, animals were placed into an

4648 in diameter round acrylic cage with lid, provided ad libitum food and water, and

465 tethered to a 100x preamplifier. All mice were housed in a 12:12 hr LD cycle and

466 acclimated to the cable tethering for $\geq 5$ days prior to recording. EEG and EMG channels

467 were sampled at $400 \mathrm{~Hz}$, high-pass filtered at $0.5 \mathrm{~Hz}$ for EEG and $10 \mathrm{~Hz}$ for EMG,

468 digitized, and then acquired using Sirena software (Pinnacle Technology).

470 Analysis: Mouse sleep was scored visually by one or two trained technicians in Sirenia

471 Sleep software (Pinnacle Technology), using raw EEG/EMG traces in 10 s epochs. Each

472 epoch was scored WAKE, NREM, or REM, and epochs with artifacts were marked for

473 exclusion in further analysis. Animals with severe movement artifacts or poor EEG

474 waveforms were excluded from all behavioural datasets. Sleep or wake bouts were

475 identified as $>30$ s of continuous sleep or wakefulness, respectively. Spectral analysis

476 was performed using custom MATLAB (Mathworks) programs, and all Fast Fourier

477 Transform spectra used 1024 or 512 point size and the Welch's power spectral density

478 estimate. Spectrograms were composed with short-time Fourier transforms with a

479 window size of 30 s, $60 \%$ overlap, and smoothened by a rolling Hann window.

\section{Stereotaxic surgeries}

482 8-12 week old male mice were anesthetized to surgical depth with a ketamine/xylazine 483 mixture (100 mg/ $\mathrm{kg}$ and $10 \mathrm{mg} / \mathrm{kg}$, respectively), and all fur was removed from the top of

484 the head. The mouse was secured into a Stoelting stereotaxis frame, and the 485 microinjector tip was placed on the Bregma and all coordinates zeroed. Small $(\sim 0.5 \mathrm{~mm})$ 
486 craniotomies were performed to allow for virus injection ( $50-300 \mathrm{nl}$ at $\sim 25 \mathrm{nl} / \mathrm{min})$.

487 Coordinates, volumes, viruses used and their sources are listed in Supplementary Table 2.

488 Post-injection, animals were allowed to heal and express viral genes for $\geq$ two weeks (for

489 projection and connectivity studies), and $\geq$ four weeks (for all behaviour and functional

490 manipulations). If sleep behaviour was measured, EEG headmounts were implanted in a

491 separate surgery. Locations of all viral injections were confirmed by post-hoc

492 immunostaining, and no animals were excluded from our analyses.

\section{Electrophysiological recordings}

495 Male mice between 5-10 weeks old were deeply anesthetized with isoflurane, and the

496 brains quickly removed and dissected in oxygenated $\left(95 \% \mathrm{O}_{2}, 5 \% \mathrm{CO}_{2}\right)$ ice-cold slicing

497 solution (2.5 mM KCl, $1.25 \mathrm{mM} \mathrm{NaH}_{2} \mathrm{PO} 4,2 \mathrm{mM} \mathrm{MgSO}_{4}, 2.5 \mathrm{mM} \mathrm{CaCl}_{2}, 248 \mathrm{mM}$

498 sucrose, $26 \mathrm{mM} \mathrm{NaHCO}_{3}$ and $10 \mathrm{mM}$ glucose $)$. Acute coronal brain slices $(250 \mu \mathrm{m})$

499 were prepared using a vibratome (VT-1200s, Leica) and then incubated in oxygenated

500 artificial cerebrospinal fluid (ACSF, $124 \mathrm{mM} \mathrm{NaCl}, 2.5 \mathrm{mM} \mathrm{KCl}, 1.25 \mathrm{mM} \mathrm{NaH}_{2} \mathrm{PO}_{4}, 2$

$501 \mathrm{mM} \mathrm{MgSO}_{4}, 2.5 \mathrm{mM} \mathrm{CaCl}_{2}, 26 \mathrm{mM} \mathrm{NaHCO}_{3}$ and $10 \mathrm{mM}$ glucose, 290-300 mOsm) at

$50228^{\circ} \mathrm{C}$ for $30 \mathrm{~min}$ and then at room temperature for $1 \mathrm{hr}$. Slices were then transferred to a

503 recording chamber, continuously perfused with oxygenated ACSF at room temperature

504 and visualized using an upright microscope (BX51WI, Olympus). Labeled cells of

505 interest were visualized using infrared differential interference contrast (IR-DIC) and

506 native fluorescence. Glass electrodes (5-8 M $\Omega)$ were filled with the following internal

507 solution (130 mM K-gluconate, $5 \mathrm{mM} \mathrm{NaCl}, 10 \mathrm{mM} \mathrm{C}_{4} \mathrm{H}_{8} \mathrm{~N}_{3} \mathrm{O}_{5} \mathrm{PNa}_{2}, 1 \mathrm{mM} \mathrm{MgCl} 2,0.2$

508 mM EGTA, 10 mM HEPES, 2 mM MgATP and 0.5 mM Na 2 GTP, pH 7.2-7.3, 300

509 mOsm). Whole-cell patch clamp recordings were obtained using a Multiclamp 700B

510 amplifier (Molecular Devices). Data were sampled at $20 \mathrm{kHz}$, low-pass filtered at $2 \mathrm{kHz}$, 511 and digitized using a Digidata 1440A (Molecular Devices).

512 For baseline spontaneous and evoked firing rate measurements, recordings were

513 performed under current clamp configuration. Baseline recordings were performed for at

514 least $30 \mathrm{~s}$ to measure spontaneous firing rate. To measure evoked firing rate, current

515 injections from -10 to $100 \mathrm{pA}$ were performed for $600 \mathrm{~ms}$. $0.5 \%$ biocytin (wt/wt) was

516 added to the internal solution to label the recorded cell. Slices were fixed post- 
517 experiment in 4\% PFA overnight, and then incubated with Alexa488-conjugated

518 streptavidin (Invitrogen, 1:2000) for $24 \mathrm{hrs,}$, and then imaged on a Zeiss 800 confocal

519 microscope. For measurement of GABA currents, voltage-clamp recordings at $-70 \mathrm{mV}$

520 were performed at ZT12-14. K-gluconate in the patch-pipette was replaced with $\mathrm{CeCl}_{2}$

521 for these recordings, $\mathrm{CeOH}$ was used to adjust the $\mathrm{pH}$ of the internal solution, and TTX

$522(1 \mu \mathrm{M})$ was added to the ACSF to block action potentials. $1 \mathrm{mM}$ GABA was delivered

523 for $5 \mathrm{~s}$ using a Picospritzer III (Parker), and GABA-evoked current was recorded. For

524 recordings of $m W a k e^{D M H}$ neurons, $\sim 30 \%$ cells exhibited spontaneous firing, and so

525 analyses of evoked responses were restricted to this subset of neurons.

526 For application of norepinephrine, orexin, acetylcholine, and histamine, current-clamp

527 recordings were performed at ZT12-14. Baseline spontaneous activity was recorded for

$52830 \mathrm{~s}$, and then for another $30 \mathrm{~s}$ following continuous application of the neurotransmitter.

529 Compounds were pre-loaded into pulled glass pipettes (3-4 $\mu \mathrm{m}$ I.D. at the tip) and

530 delivered at the recorded cell using a Picospritzer III (Parker). The compounds and

531 concentrations used were as follows: norepinephrine $(100 \mu \mathrm{M})$, acetylcholine chloride (1

$532 \mathrm{mM})$, orexin-A $(300 \mathrm{~nm})$, and histamine $(20 \mu \mathrm{M})$. For synaptically isolating recorded

533 cells, ion channel blockers (20 $\mu \mathrm{M}$ CNQX; $50 \mu \mathrm{M}$ AP5; $10 \mu \mathrm{M}$ Picrotoxin) were added to

534 the ACSF perfusion. For quantification of firing rates for cells reaching plateau potential,

535 the maximum firing rate observed for any cell following application of the relevant

536 neurotransmitter was used.

537 For optogenetic experiments, AAV-DIO-hChR2 (Supplementary Table 2) was

538 injected into the DMH of $m W k^{\left(\mathrm{Cre}^{+}\right)} ; \mathrm{TH}-\mathrm{GFP}$ mice. Acute slices were prepared 3

539 weeks post-viral injection. Current-clamp and voltage-clamp recordings were performed

540 from norepinephrine-expressing cells in the locus coeruleus $\left(\mathrm{TH}^{+}\right)$, in the presence of

541 optogenetic activation of ChR2-expressing terminals in the LC. Slices were exposed to

542 blue light $(480 \mathrm{~nm})$ from the upper lens for $2 \mathrm{~s}$ at different stimulation frequencies $(5,10$,

54320 , and $50 \mathrm{~Hz}$ ) triggered by the Digidata 1440A.

\section{Immunohistochemistry}

546 Mice were deeply anesthetized with a ketamine/xylazine mixture then fixed by

547 transcardial perfusion with 4\% paraformaldehyde (PFA). Brains were subsequently 
548 drop-fixed in 4\% PFA for 24-48 $\mathrm{hr}$ and transferred into 1x PBS before being sectioned at

$54940 \mu \mathrm{m}$ thickness using a vibratome (VT1200S, Leica). Free-floating sections were

550 washed in $1 \mathrm{x}$ PBS, blocked for $1 \mathrm{hr}$ in blocking buffer (PBS containing $0.25 \%$ Triton-X-

551100 and $5 \%$ normal goat serum or normal donkey serum), then incubated with rabbit anti-

552 HDC (Progen, 16045, 1:800), goat anti-ChAT (Millipore, AB144P, 1:250), rabbit anti-

553 orexin A (Abcam, AB6214, 1:500), chicken anti-TH (Abcam, AB76442, 1:1000), or rat

554 anti-HA (Roche $11867423001,1: 250$ ) in blocking buffer at $4^{\circ} \mathrm{C}$ overnight. The

555 following day, slices were washed with PBST (PBS and 0.1\% Tween-20), then incubated

556 with Alexa 488 anti-rabbit (ThermoFisher, A-11008, 1:2500-1:5000), Alexa 568 anti

557 rabbit (ThermoFisher, A-11011, 1:2500), Alexa 568 anti-goat (ThermoFisher, A11057,

558 1:2000), Alexa 488 anti-rat (ThermoFisher A-11006, 1:2000), or Alexa 488 anti-chicken

559 (ThermoFisher, A-11039, 1:2000) secondary antibodies for 2.5 hrs in blocking buffer.

560 Brain sections were then washed in PBST, incubated in DAPI (1:2000, Millipore) for 5

$561 \mathrm{~min}$, then washed in PBS. Sections were mounted on slides using VECTASHIELD

562 HardSet Mounting Medium (Vector Laboratories, USA). In all tdTomato images, native

563 fluorescence of tdTomato was visualized. Images were acquired using a Zeiss LSM800

564 confocal microscope under 10x-63x magnification.

\section{In situ hybridization}

568 ISH for $m$ Wake was performed as previously described ${ }^{4}$. RNAScope ISH was performed 569 using the RNAscope 2.5 Chromogenic Assay and the BaseScope ${ }^{\mathrm{TM}}$ Detection Reagent

570 Kit according to the manufacturer's instructions (Advanced Cell Diagnostics (ACD) $)^{44}$.

571 Target probes were designed to exon 4 of $m$ Wake. Mouse brains were dissected and fresh

572 frozen in Tissue-Tek O.C.T. (VWR) and cryosectioned at $10 \mu \mathrm{m}$. Sections were treated

573 with hydrogen peroxide and protease, before hybridization to the custom $m$ Wake probe

574 and subsequent amplification. Signal was detected by chromogenic reaction with

575 BaseScope ${ }^{\mathrm{TM}}$ Fast RED, and sections counterstained with hematoxylin. Images were

576 acquired on a Keyence BZ-X700 microscope (Keyence) under 10x brightfield

577 illumination. Fluorescent in situ hybridization (FISH) was performed with the

578 RNAscope ${ }^{\mathrm{TM}}$ Fluorescent Multiplex Assay (ACD), using probes targeting tdTomato and

579 Slc32al (VGat) or Slc17a6 (VGlut2) mRNA. Preparation of tissue sections was 
580 performed as above, followed by simultaneous hybridization to both probes. Probe

581 binding was indicated by deposition of target-specific fluorophores at each location via

582 TSA Plus Fluorescence kit (PerkinElmer), and sections were then counterstained with

583 DAPI. Sections were imaged on a Zeiss LSM880 confocal at 20x and 63x.

\section{Designer Receptors Exclusively Activated by Designer Drugs (DREADDs)}

586 DREADD receptors coupled to either Gq or Gi were expressed in a Cre-dependent

587 fashion in $\mathrm{mWake}^{+}$neurons of $m \mathrm{Wake}^{(\mathrm{Cre} /+)}$ mice, either locally in the DMH, via

588 stereotaxic injection of a viral vector (AAV-DIO-DREADD-Gq or AAV-DIO-

589 DREADD-Gi) (Supplementary Table 2), or globally via crossing with a transgenic

590 effector mouse line (B6N;129-CAG-LSL-HA-hM4Di-mCitrine, "LSL-Gi"). Clozapine $N$ -

591 oxide (CNO) (SigmaAldrich) was prepared as a stock solution of $50 \mathrm{mg} / \mathrm{ml}$ in DMSO,

592 and then freshly diluted to $0.1 \mathrm{mg} / \mathrm{ml}$ in sterile PBS before IP injection. Solution clarity

593 was monitored throughout dosing, and the solution was warmed to $37^{\circ} \mathrm{C}$ if precipitates

594 were observed. Vehicle control was prepared as sterile saline $+0.01 \%$ DMSO. All

595 injections occurred at the same ZT/CT time within each experiment, and all animals were

596 treated with vehicle or $\mathrm{CNO}$ each day in a cross-over design, with $\geq 2$ days recovery

597 between experimental recording days. To control for CNO activity on its own, 1 or 3

$598 \mathrm{mg} / \mathrm{kg} \mathrm{CNO}$ were IP injected into sham-injected $\mathrm{mWake} \mathrm{Cre}^{(+)}$mice and locomotion and

599 EEG data were assessed.

600

601 Statistical analysis

602 Statistical analyses were performed in Prism 7 and 8 (Graphpad). For comparisons of

603 two groups of normally distributed data, unpaired Student t-tests were used; if these

604 comparisons were before and after treatment of the same animals or cells, paired t-tests

605 were used instead. For comparisons of two groups of non-normally distributed data,

606 Mann Whitney U tests were performed, with a Holm-Bonferroni correction, if required

607 for multiple comparisons. For multiple comparisons of normally distributed data with 2

608 factors, 2 way ANOVAs were performed (with repeated measures, if applicable),

609 followed by post-hoc Sidak tests. For multiple comparisons of non-normally distributed

610 data, Kruskall-Wallis tests were performed with post-hoc Dunn's tests. 
bioRxiv preprint doi: https://doi.org/10.1101/2020.03.12.989921; this version posted May 30, 2020. The copyright holder for this preprint (which was not certified by peer review) is the author/funder. All rights reserved. No reuse allowed without permission.

611

612 


\author{
613 References \\ 6141 Allada, R., Emery, P., Takahashi, J. S. \& Rosbash, M. Stopping time: the genetics \\ 615 of fly and mouse circadian clocks. Annual review of neuroscience 24, 1091-1119 \\ $616 \quad$ (2001). \\ 6172 Mohawk, J. A., Green, C. B. \& Takahashi, J. S. Central and peripheral circadian \\ 618 clocks in mammals. Annual review of neuroscience 35, 445-462 (2012). \\ 6193 Scammell, T. E., Arrigoni, E. \& Lipton, J. O. Neural Circuitry of Wakefulness \\ 620 and Sleep. Neuron 93, 747-765, doi:10.1016/j.neuron.2017.01.014 (2017). \\ 6214 Liu, S. et al. WIDE AWAKE mediates the circadian timing of sleep onset. \\ $622 \quad$ Neuron 82, 151-166, doi:10.1016/j.neuron.2014.01.040 (2014). \\ 6235 Tabuchi, M. et al. Clock-Generated Temporal Codes Determine Synaptic \\ 624 \\ 625 \\ 626 \\ 627 \\ 628 \\ 629 \\ 630 \\ 631 \\ 632 \\ 633 \\ 634 \\ 635 \\ 636 \\ 637 \\ 638 \\ 639 \\ 640 \\ 641 \\ 642 \\ 643 \\ 644 \\ 645 \\ 646 \\ 647 \\ 648 \\ 649 \\ 650 \\ 651 \\ 652 \\ 653 \\ 654 \\ 655 \\ 656 \\ 657 \\ $658 \quad 16$ \\ 659 \\ Plasticity to Control Sleep. Cell 175, 1213-1227 e1218, \\ doi:10.1016/j.cell.2018.09.016 (2018). \\ 6 Zhang, S. et al. Nmf9 Encodes a Highly Conserved Protein Important to \\ Neurological Function in Mice and Flies. PLoS Genet 11, e1005344, \\ doi:10.1371/journal.pgen.1005344 (2015). \\ 7 Pfaff, D., Ribeiro, A., Matthews, J. \& Kow, L. M. Concepts and mechanisms of \\ generalized central nervous system arousal. Ann N Y Acad Sci 1129, 11-25, \\ doi:10.1196/annals.1417.019 (2008). \\ 8 Homanics, G. E. et al. Mice devoid of gamma-aminobutyrate type A receptor \\ beta3 subunit have epilepsy, cleft palate, and hypersensitive behavior. \\ Proceedings of the National Academy of Sciences of the United States of America \\ 94, 4143-4148, doi:10.1073/pnas.94.8.4143 (1997). \\ 9 Glick, S. D., Zimmerberg, B. \& Greenstein, S. Individual differences among mice \\ in normal and amphetamine-enhanced locomotor activity: relationship to \\ behavioral indices of striatal asymmetry. Brain research 105, 362-364, \\ doi:10.1016/0006-8993(76)90436-4 (1976). \\ 10 Tilley, M. R. \& Gu, H. H. Dopamine transporter inhibition is required for \\ cocaine-induced stereotypy. Neuroreport 19, 1137-1140, \\ doi:10.1097/WNR.0b013e3283063183 (2008). \\ 11 Bell, B. J., Wang, A. W., Kim, D. W., Blackshaw, S. \& Wu, M. N. \\ Characterization of $m$ Wake expression in the murine brain. bioRxiv doi: \\ https://doi.org/10.1101/2020.05.25.114363 (2020). \\ 12 Bunger, M. K. et al. Mop3 is an essential component of the master circadian \\ pacemaker in mammals. Cell 103, 1009-1017, doi:10.1016/s0092- \\ 8674(00)00205-1 (2000). \\ 13 Furuta, Y., Lagutin, O., Hogan, B. L. \& Oliver, G. C. Retina- and ventral \\ forebrain-specific Cre recombinase activity in transgenic mice. Genesis 26, 130 - \\ $132(2000)$. \\ 14 Chou, T. C. et al. Critical role of dorsomedial hypothalamic nucleus in a wide \\ range of behavioral circadian rhythms. The Journal of neuroscience : the official \\ journal of the Society for Neuroscience 23, 10691-10702 (2003). \\ 15 Aston-Jones, G., Chen, S., Zhu, Y. \& Oshinsky, M. L. A neural circuit for \\ circadian regulation of arousal. Nature neuroscience 4, 732-738, \\ doi:10.1038/89522 (2001). \\ 16 Deurveilher, S. \& Semba, K. Indirect projections from the suprachiasmatic \\ nucleus to major arousal-promoting cell groups in rat: implications for the
}




\begin{tabular}{|c|c|c|}
\hline $\begin{array}{l}660 \\
661\end{array}$ & & $\begin{array}{l}\text { circadian control of behavioural state. Neuroscience 130, 165-183, } \\
\text { doi:10.1016/j.neuroscience.2004.08.030 (2005). }\end{array}$ \\
\hline $\begin{array}{l}662 \\
663\end{array}$ & 17 & $\begin{array}{l}\text { Krashes, M. J. et al. Rapid, reversible activation of AgRP neurons drives feeding } \\
\text { behavior in mice. } J \text { Clin Invest 121, 1424-1428, doi:10.1172/JCI46229 (2011). }\end{array}$ \\
\hline $\begin{array}{l}064 \\
665 \\
666\end{array}$ & 18 & $\begin{array}{l}\text { Kim, D. W. et al. Single cell RNA-Seq analysis identifies molecular mechanisms } \\
\text { controlling hypothalamic patterning and differentiation. bioRxiv doi: } \\
\text { https://doi.org/10.1101/657148 }(2020) \text {. }\end{array}$ \\
\hline 667 & 19 & Saper, C. B. \& Fuller, P. M. Wake-sleep circuitry: an overview. Curr Opin \\
\hline & & Neurobiol 44, 186-192, doi:10.1016/j.conb.2017.03.021 (2017). \\
\hline 99 & 20 & Eban-Rothschild, A., Appelbaum, L. \& de Lecea, L. Neuronal Mechanisms for \\
\hline & & Sleep/Wake Regulation and Modulatory Drive. Neuropsychopharmacology 43, \\
\hline & & 937-952, doi:10.1038/npp.2017.294 (2018). \\
\hline $\begin{array}{l}6 \\
6\end{array}$ & 21 & $\begin{array}{l}\text { Jones, B. E. Arousal and sleep circuits. Neuropsychopharmacology 45, 6-20, } \\
\text { doi:10.1038/s41386-019-0444-2 (2020). }\end{array}$ \\
\hline 74 & 22 & Blanco-Centurion, C., Gerashchenko, D. \& Shiromani, P. J. Effects of saporin- \\
\hline & & induc \\
\hline 6 & & The Journal of neuroscience : the official journal of the Society for Neuroscience \\
\hline & & $27,14041-14048$ (2007). \\
\hline & 23 & Tsunematsu, T. et al. Acute optogenetic silencing of orexin/hypocretin neurons \\
\hline & & induces slow-wave sleep in mice. J. Neurosci. 31, 10529-10539, \\
\hline & & doi:10.1523/JNEUROSCI.0784-11.2011 (2011). \\
\hline & 24 & Venner, A. et al. Reassessing the Role of Histaminergic Tuberomammillary \\
\hline & & Neurons in Arousal Control. The Journal of neuroscience : the official journal of \\
\hline & & the Society for Neuroscience 39, 8929-8939, doi:10.1523/JNEUROSCI.1032- \\
\hline & & $19.2019(2019)$ \\
\hline & 25 & Yu, X. et al. Genetic lesioning of histamine neurons increases sleep-wake \\
\hline & & fragmentation and reveals their contribution $t$ \\
\hline & & Sleep 42, doi:10.1093/sleep/zsz031 (2019). \\
\hline & 26 & Carter, M. E. et al. Tuning arousal with optogenetic modulation of locus \\
\hline & & coeruleus neurons. Nature neuroscience 13, 1526-1533, doi:10.1038/nn.2682 \\
\hline & & $(2010)$ \\
\hline 6 & 27 & Anaclet, C. et al. Basal forebrain control of wakefulness and cortical rhythms. \\
\hline & & Nat. Commun. 6, 8744, doi:10.1038/ncomms974 \\
\hline & 28 & Xu, M. et al. Basal forebrain circuit for sleep-wake control. Nature neuroscience \\
\hline & & 18, 1641-1647, doi:10.1038/nn.4143 (2015). \\
\hline & 29 & Herrera, C. G. et al. Hypothalamic feedforward inhibition of thalamocortical \\
\hline & & network controls arousal and consciousness. Nature neu \\
\hline & & doi:10.1038/nn.4209 (2016). \\
\hline cro & 30 & Venner, A., Anaclet, C., Broadhurst, R. Y., Saper, C. B. \& Fuller, P. M. A Novel \\
\hline & & Population of Wake-Promoting GABAergic Neurons in the Ventral Lateral \\
\hline 100 & & Hypothalamus. Curr. Biol. 26, 2137-2143, doi:10.1016/j.cub.2016.05.078 (2016). \\
\hline 70 & 31 & Kroeger, D. et al. Cholinergic, Glutamatergic, and GABAergic Neurons of the \\
\hline & & Pedunculopontine Tegmental Nucleus Have Distinct Effects on Sleep/Wake \\
\hline & & Behavior in Mice. J. Neurosci. 37, 1352-1366, doi:10.1523/JNEUROSCI.1405- \\
\hline & & \\
\hline
\end{tabular}


32 Zhu, H. et al. Cre-dependent DREADD (Designer Receptors Exclusively Activated by Designer Drugs) mice. Genesis 54, 439-446, doi:10.1002/dvg.22949 (2016).

33 Mistlberger, R. E. Circadian regulation of sleep in mammals: role of the suprachiasmatic nucleus. Brain Res Brain Res Rev 49, 429-454 (2005).

34 Cheng, M. Y. et al. Prokineticin 2 transmits the behavioural circadian rhythm of the suprachiasmatic nucleus. Nature 417, 405-410, doi:Doi 10.1038/417405a (2002).

35 Kramer, A. et al. Regulation of daily locomotor activity and sleep by hypothalamic EGF receptor signaling. Science 294, 2511-2515 (2001).

36 Kraves, S. \& Weitz, C. J. A role for cardiotrophin-like cytokine in the circadian control of mammalian locomotor activity. Nature neuroscience 9, 212-219, doi:10.1038/nn1633 (2006).

37 Pevet, P. \& Challet, E. Melatonin: both master clock output and internal timegiver in the circadian clocks network. J Physiol Paris 105, 170-182, doi:10.1016/j.jphysparis.2011.07.001 (2011).

$38 \mathrm{Yu}$, X. et al. Circadian factor BMAL1 in histaminergic neurons regulates sleep architecture. Current biology : $C B$ 24, 2838-2844, doi:10.1016/j.cub.2014.10.019 (2014).

39 Fuller, P. M., Sherman, D., Pedersen, N. P., Saper, C. B. \& Lu, J. Reassessment of the structural basis of the ascending arousal system. The Journal of comparative neurology 519, 933-956, doi:10.1002/cne.22559 (2011).

40 Coull, J. T. Neural correlates of attention and arousal: insights from electrophysiology, functional neuroimaging and psychopharmacology. Prog Neurobiol 55, 343-361, doi:10.1016/s0301-0082(98)00011-2 (1998).

41 Wu, Y. E., Pan, L., Zuo, Y., Li, X. \& Hong, W. Detecting Activated Cell Populations Using Single-Cell RNA-Seq. Neuron 96, 313-329 e316, doi:10.1016/j.neuron.2017.09.026 (2017).

42 Stuart, T. et al. Comprehensive Integration of Single-Cell Data. Cell 177, 18881902 e1821, doi:10.1016/j.cell.2019.05.031 (2019).

43 Lein, E. S. et al. Genome-wide atlas of gene expression in the adult mouse brain. Nature 445, 168-176, doi:10.1038/nature05453 (2007).

44 Wang, F. et al. RNAscope: a novel in situ RNA analysis platform for formalinfixed, paraffin-embedded tissues. J Mol Diagn 14, 22-29, doi:10.1016/j.jmoldx.2011.08.002 (2012). 


\section{Acknowledgements}

746 We thank A. Meredith and B. McNally for advice on SCN patch-clamp recordings and S.

747 Hattar for assistance with EEG recordings. We thank B. Hamilton, A. Jackson, A.

748 McCallion, and Y. Furuta for sharing mouse strains. We thank the Transcriptomics and

749 Deep Sequencing Core for scRNA sequencing and the Ross Flow Cytometry Core flow

750 sorting. We thank members of the Wu Lab for discussion. This work was supported by

751 NIH grants R01NS094571 and R01NS079584. (M.N.W.) and a NINDS Center grant

752 NS05027 for machine shop work.

753

754 Author Contributions

755 B.J.B. and M.N.W. conceived the project, with input from J.Y.C. and S.B. B.J.B.

756 performed most or all mouse genetics, behavioural experiments, EEG recordings,

757 expression analyses, viral injections, and rabies tracing. Q.L.* performed all

758 electrophysiology and optogenetic experiments and assisted with EEG analyses. D.W.K.

759 performed scRNA-Seq analysis. S.S.L. performed viral injections and immunostaining.

760 Q.L. assisted with mouse genetics and generation of the $m W a k e^{-}$allele. I.D.B. assisted

761 with EEG analyses. A.A.W. and J.L.B. assisted with expression analyses. A.J.C.

762 assisted with viral injections. H.I. assisted with mouse genetics. B.J.B. and M.N.W.

763 wrote the manuscript, with feedback from all authors. 


\section{Figure Legends}

\section{Figure 1 | mWAKE inhibits arousal and SCN firing at night}

768 a, $m$ Wake mRNA detected by BaseScope ISH demonstrates expression in the SCN core

769 (solid and dashed lines denote SCN and core region, respectively). Higher magnification

770 inset shows representative $m$ Wake mRNA expression in cells. Scale bar applies to both

771 images and denotes $200 \mu \mathrm{m}$ and $50 \mu \mathrm{m}$ for the entire image and inset, respectively. b,

772 Schematic showing genomic structure of the $m$ Wake locus and CRISPR/Cas9-mediated

773 insertion of an in-frame stop codon in exon 4 in the $m W a k e^{-}$mutation. c, Relative mRNA

774 level for $m$ Wake, determined by qPCR, in $m W a k e^{(--)}$vs WT littermate control

775 hypothalami (normalized to 1.0) ( $\mathrm{n}=3$ replicates). $\mathbf{d}$, Representative images (of 4

776 replicates each) of ISH using a digoxigenin-labeled $m$ Wake RNA probe in the SCN of

$777 m$ Wake ${ }^{(--)}$vs WT mice. Scale bar denotes $500 \mu \mathrm{m}$. e, Vigilance state (\% per $2 \mathrm{hr}$ bin)

778 determined by EEG recordings for $m W_{a k e}^{(--)}(\mathrm{n}=8)$ vs WT littermate control $(\mathrm{n}=6)$ mice

779 under DD conditions. “"”” and “\#” denote $P<0.01$ and $P<0.001$, respectively. f, Profile of

780 locomotor activity (defined by beam-breaks) over $24 \mathrm{hrs}$ for $m \operatorname{Wake}^{(+/)}(\mathrm{n}=19$, grey),

$781 m \operatorname{Wake}^{(+/)}(\mathrm{n}=22$, black $), m W a k e^{(-/)}(\mathrm{n}=19$, red $)$, and $m W_{a k e^{(N m f 9 /-)}}(\mathrm{n}=9$, cyan) mice

782 under DD conditions. g, Total locomotor activity (total number of beams broken along X

783 and Y axes) from CT0-12 and CT12-24 from the mice described in (f). h, Startle

784 response (Vavg) measured in the first $100 \mathrm{~ms}$ following a 100, 110, or $120 \mathrm{~dB}$ tone for

$785 m$ Wake $^{(+/)}$(grey, $\left.\mathrm{n}=10\right)$ vs $m W_{a k e}^{(--)}$mice (red, $\left.\mathrm{n}=10\right)$ at CT12-14. The average of 5

786 responses is shown. i, Schematic showing genomic structure of the $m$ Wake locus and

787 replacement of exon 5 with a tdTomato-P $2 A$-Cre cassette in the $m$ Wake ${ }^{(\text {Cre })}$ line. $\mathbf{j}$,

788 Native tdTomato fluorescence in the SCN of a $\mathrm{mWake} \mathrm{Cre}^{(+)}$mouse (solid and dashed

789 lines denote SCN and core region, respectively). Scale bar represents $200 \mu \mathrm{m}$. k,

790 Representative membrane potential traces from whole-cell patch clamp recordings of

$791 m W a k e^{S C N}$ neurons from $m W_{a k e}^{(C r e /+)}$ (grey, top) and $m W a k e^{(C r e / C r e)}$ (red, bottom) slices

792 at ZT0-2 and ZT12-14. 1, Spontaneous mean firing rate for $m W_{a k e}^{+} \mathrm{SCN}$ neurons at

793 ZT0-2 and ZT12-14 from $m \operatorname{Wake}^{(\mathrm{Cre} /+)}(\mathrm{n}=21$ and $\mathrm{n}=22)$ vs $m$ Wake $^{(\mathrm{Cre} / \mathrm{Cre})}(\mathrm{n}=24$ and

$794 \mathrm{n}=20$ ) mice. $\mathbf{m}$, Representative traces of voltage-clamp recordings of $m W a k e^{S C N}$ neurons

795 of $m W_{a k e^{(\mathrm{Cre} /+)}}$ vs $m W a k e^{(\mathrm{Cre} / \mathrm{Cre})}$ at ZT12-14. Timing of GABA (1 mM) application is

796 shown. n, GABA-evoked current in $m W_{a k} e^{S C N}$ neurons of $m W_{a k}^{(C r e /+)}(\mathrm{n}=18$, grey) vs 
$797 m \operatorname{Wake}^{(\mathrm{Cre} / \mathrm{Cre})}(\mathrm{n}=20$, red $)$ at ZT12-14. For panels (I) and (n), $\mathrm{n}$ represents individual

798 cells from 4 animals for each condition. In this figure and throughout, error bars

799 represent SEM and “*”, “**”, and “***” denote $P<0.05, P<0.01$, and $P<0.001$,

800 respectively.

802 Figure $2 \mid$ mWake $e^{D M H}$ neurons drive arousal

803 a, mWake mRNA detected by Basescope ISH reveals expression in the dorsomedial

804 hypothalamus (DMH). Dashed lines indicate DMH region, and inset shows

805 representative $m$ Wake mRNA expression in cells. Scale bar applies to both images and

806 denotes $200 \mu \mathrm{m}$ and $50 \mu \mathrm{m}$ for the entire image and inset, respectively. b, Native

807 tdTomato fluorescence in the DMH of a $m W_{a k e}^{\left(\mathrm{Cre}^{+}\right)}$mouse (dashed lines denote DMH

808 region). Scale bar represents $200 \mu \mathrm{m}$. c, Representative membrane potential traces from

809 whole-cell patch clamp recordings of $m W_{a k e^{D M H}}$ neurons in $\mathrm{mWake}^{\text {(Cre/+) }}$ (grey, top) and

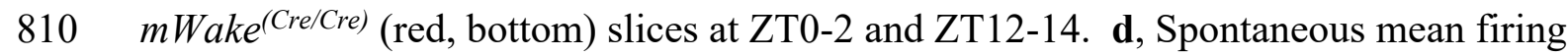

811 rate for $m W a k e^{D M H}$ neurons at ZT0-2 and ZT12-14 from $m W_{a k e}^{(\mathrm{Cre} /+)}(\mathrm{n}=19$ and n=14) vs

$812 m$ Wake $^{(\mathrm{Cre} / \mathrm{Cre})}(\mathrm{n}=16$ and $\mathrm{n}=12)$ mice. $\mathrm{n}$ represents individual cells from $\geq 4$ animals. e,

813 Schematic showing bilateral injections of AAV viral vector containing Cre-recombinase

814 and eGFP (AAV-Cre), or eGFP alone (AAV-Sham) into the DMH of $m$ Wake (floxflox)

815 mice. f, Representative image of eGFP fluorescence expression in the DMH following

816 AAV-Cre injection described in (e). Scale bar denotes $200 \mu \mathrm{m}$. g, Total locomotor

817 activity (total number of beams broken along $\mathrm{X}$ and $\mathrm{Y}$ axis) during CT0-4 vs CT12-16

818 under DD conditions for $m$ Wake $e^{(f l o x / f l o x)}$ mice before ("pre", grey) and after ("post", green)

819 DMH injection of AAV-Sham $(n=6)$ or AAV-Cre $(n=9)$. h, Schematic showing bilateral

820 injections of AAV-DIO-DREADD-Gq into the DMH of $m W_{a k e^{(C r e /+)}}$ mice. i,

821 Representative image of mCherry fluorescence expression in the DMH of $m$ Wake(Cre/+)

822 following AAV-DIO-DREADD-Gq injection described in (h). Scale bar denotes 200

$823 \mu \mathrm{m}$. j, Total locomotor activity (total number of beams broken along $\mathrm{X}$ and $\mathrm{Y}$ axis) of

$824 m \mathrm{Wake}^{(\mathrm{Cre} /+)}$ mice with bilateral injections of AAV-DIO-DREADD-Gq into the DMH in

825 the $4 \mathrm{hrs}$ following IP injection of vehicle vs CNO $(1 \mathrm{mg} / \mathrm{kg})$ at CT $8(\mathrm{n}=6)$. $\mathbf{k}$,

826 Representative short-time Fourier transform spectrograms of $8 \mathrm{hrs}$ of recorded EEG

827 activity, starting after IP injection at ZT6 of vehicle alone (above) or $1 \mathrm{mg} / \mathrm{kg} \mathrm{CNO}$ 
828 (below), from $m W a k e^{\left(\text {Cre/+) }^{+}\right.}$mice injected with AAV-DIO-DREADD-Gq bilaterally into

829 the DMH. Power density is represented by the color-scheme and deconvoluted by

830 frequency on the $y$-axis, over time on the x-axis. I, Amount of wakefulness derived from

831 EEG plotted as \% time in $1 \mathrm{hr}$ bins following IP injection of vehicle (grey) or $1 \mathrm{mg} / \mathrm{kg}$

832 CNO (red) $(n=4)$ at ZT6. "3v" denotes third ventricle.

834 Figure 3 | Characterization of $\boldsymbol{m} W a k e^{D M H}$ neurons and their interaction with

835 neuromodulatory systems

836 a, UMAP (Uniform Manifold Approximation and Projection) plot showing distribution

837 of $m W_{a k e} e^{+}$neurons across hypothalamic nuclei, as determined by single-cell expression

838 profiling. 11 clusters are defined, for SCN, DMH, POA (pre-optic area), TMN

839 (tuberomammillary nucleus), ZI (zona incerta), and VMH (ventromedial hypothalamus)

840 regions. "Gal ${ }^{+}$" and " $\mathrm{Cck}^{+}$" refer to Galanin ${ }^{+}$and Cholecystokinin'. b, Bar graph

841 showing proportions of GABAergic and glutamatergic $m W_{a k e}^{+}$neurons for each scRNA-

842 Seq neuronal cluster. c, Bar graph showing distribution of expression for norepinephrine,

843 dopamine, acetylcholine, histamine and orexin receptors identified in DMH $m W a k e^{+}$

844 neurons (cluster 4). d, Schematic showing patch-clamp recordings of $m W a k e^{D M H}$ neurons

845 from $m \mathrm{Wake}^{(\mathrm{Cre} /+)}$ mice, following application of norepinephrine, orexin, or

846 acetylcholine. e, Representative membrane potential traces from recordings described in

847 (d) following administration of norepinephrine (top, red), orexin (middle, green), or

848 acetylcholine (bottom, blue) in the presence of synaptic blockers at ZT12-14. f,

849 Spontaneous mean firing rate of $m W a k e^{D M H}$ neurons following application of $100 \mu \mathrm{M}$

850 norepinephrine ( $\mathrm{n}=23$ and 12 cells), $300 \mathrm{nM}$ orexin- $\mathrm{A}(\mathrm{n}=8$ and 7 cells), or $1 \mathrm{mM}$

851 acetylcholine chloride ( $\mathrm{n}=8$ and 10 cells) at ZT12-14 in the absence ("- blockers") or

852 presence ("+ blockers") of $20 \mu \mathrm{M}$ CNQX, $50 \mu \mathrm{M}$ AP5, and $10 \mu \mathrm{M}$ picrotoxin. The

853 neurons recorded in $\mathbf{f}$ were derived from 3-6 animals. g, Schematic showing AAV-DIO-

$854 \mathrm{hChR} 2$ injected into the DMH of $m W a k e^{(\mathrm{Cre} /+)} ; \mathrm{TH}-G F P$ mice unilaterally, and whole-cell

855 patch clamp recordings of $N E^{L C}$ neurons, following $480 \mathrm{~nm}$ blue-light stimulation. $\mathbf{h}$ and

856 i, Representative excitatory post-synaptic currents (EPSCs) (h) or action potentials (APs)

857 (i) from $N E^{L C}$ neurons, triggered by optogenetic stimulation of $m W a k e^{D M H}$ neuron

858 terminals at $5 \mathrm{~Hz}$. Blue lines indicate timing of blue-light pulses. j, Fidelity (\%) of $N E^{L C}$ 
859 neuron EPSCs (dark blue) and APs (light blue) triggered by optogenetic stimulation of

$860 m W a k e^{D M H}$ neuron terminals. A total of $7 N E^{L C}$ cells from 3 independent virally-injected

$861 \mathrm{mWake}{ }^{(\mathrm{Cre} /+)} ; \mathrm{TH}-\mathrm{GFP}$ mice were recorded, of which 4 exhibited optogenetically-

862 triggered responses.

864 Figure 4 | mWAKE defines a network critical for arousal

865 a, Jitter plot showing behavioural classification of arousal level for $m W a k e^{(\text {Cre/+) }} ; L S L-G i$

866 mice $90 \mathrm{~min}$ following injection of vehicle or $0.3 \mathrm{mg} / \mathrm{kg} \mathrm{CNO}(\mathrm{n}=12)$ at ZT12. b, Startle

867 response (Vmax) measured in the first $100 \mathrm{~ms}$ following a $120 \mathrm{~dB}$ tone for $\mathrm{mWake} \mathrm{Cre}^{\text {+ })}$;

$868 L S L-G i$ mice $2 \mathrm{hr}$ following injection of vehicle (grey) or $0.3 \mathrm{mg} / \mathrm{kg}$ CNO (magenta)

$869(\mathrm{n}=5)$ at CT12-14. c, Representative short-time Fourier transform spectrograms of $12 \mathrm{hrs}$ 870 of recorded EEG activity from $m W a k e^{\left(\mathrm{Cre}^{++}\right)} ; L S L-G i$ mice after IP injection at ZT10 of

871 vehicle alone (above) or $0.3 \mathrm{mg} / \mathrm{kg} \mathrm{CNO}$ (below). d, Representative EEG traces for a 30

$872 \mathrm{~s}$ window (above) and short-time Fourier transform spectrograms (below) starting 150

$873 \mathrm{mins}$ after injection of vehicle (left) or $0.3 \mathrm{mg} / \mathrm{kg} \mathrm{CNO}$ (right) at ZT10. The dashed red

874 box indicates the time window used for spectral and amplitude analysis in (e) and (f). e,

875 Welch's power spectral density estimates as a percentage of total EEG power across 10

$876 \mathrm{~min}$, averaged across multiple EEG traces. $m \mathrm{Wake}^{(\mathrm{Cre} /+)}$; $L S L-G i$ injected with vehicle

877 (grey) or $0.3 \mathrm{mg} / \mathrm{kg} \mathrm{CNO}$ (magenta) $(\mathrm{n}=4)$. Inset shows a plot of delta-band power as a

878 percentage of total EEG power. f, EEG trace amplitude (plotted as normalized root mean 879 square (RMS)) for the animals described in (e).

Extended Data Figure 1 | Circadian and EEG-related phenotypes of $m$ Wake mutant

883 a, Schematic showing domain structure of mWAKE in mice and humans, compared to

884 Drosophila WAKE. Percentage similarity of mouse and human mWAKE, compared to

885 fly WAKE is shown. b, Representative double-plotted actograms of wheel running 886 activity for $m W a k e^{(+/+)}$(left) vs $m W_{a k e}^{(-/)}$mice (right), covering 14 days of LD then 14

887 days of free-running in DD. Activity plotted as number of wheel revolutions in $10 \mathrm{~min}$

888 bins. c, Locomotor period length $(\tau)$ during the DD period for $m \operatorname{Wake}^{(+/+)}(\mathrm{n}=8)$ and

$889 m \operatorname{Wake}^{(--)}(\mathrm{n}=8)$ mice. d, Vigilance state $(\%$ per $2 \mathrm{hr}$ bin $)$ determined by EEG recordings 
890 for $m$ Wake $^{(--)}(\mathrm{n}=8)$ vs WT littermate control $(\mathrm{n}=6)$ mice under LD conditions. The mice

891 in panel (d) are the same as in Fig. 1e. e, Hypnograms (top) and short-time Fourier

892 transform spectrograms (bottom) over $24 \mathrm{hrs}$ in DD showing example of prolonged wake

893 bout in $m W a k e^{(-/)}$mice, compared to $m W a k e^{(+/+)}$control. Power density is represented

894 by the color-scheme and deconvoluted by frequency on the y-axis, over time on the x-

895 axis. f, Wakefulness bout length distribution over a $24 \mathrm{hr}$ period expressed as a \% of

896 total wakefulness, for $m W a k e^{(++)}$vs $m W a k e^{(-/)}$mice. Dashed line highlights the presence

897 of $>6 \mathrm{hr}$ continuous bouts of wakefulness, which are observed in some $m$ Wake $e^{(--)}$

898 mutants and never in controls.

900 Extended Data Figure $2 \mid$ mWake mutants are hyperactive at night

901 a, Schematic of the beam-break arena used to measure locomotion, with infrared red

902 beams forming a grid spaced at 0.5 inch intervals across the floor of the cage. b, Profile

903 of locomotor activity (defined by beam breaks) over $24 \mathrm{hrs}$ for $m W_{a k e^{(++)}}$(n=19, grey),

$904 m \operatorname{Wake}^{(--)}(\mathrm{n}=19$, red $)$, and $m W_{a k e}^{(N m f 9 /-)}(\mathrm{n}=10$, cyan $)$ mice under LD conditions. c,

905 Total locomotor activity (total number of beams broken along $\mathrm{X}$ and $\mathrm{Y}$ axes) from the

906 mice in (b) at CT0-12 and CT12-24. Note that these data are from the same mice as in

907 Fig. 1f. d, Startle response (Vavg) following a 100, 110, or $120 \mathrm{~dB}$ tone for $m$ Wake ${ }^{(+/+)}$

908 (grey, $\mathrm{n}=10$ ) vs $m W a k e^{(--)}$mice (red, $\mathrm{n}=10$ ) at CT0-2. Note that these data are from the

909 same mice as in Fig. 1h. e and $\mathbf{g}$, Distance $(\mathrm{cm})$ traveled in five min bins in an open-field

910 test, across $3 \mathrm{hrs}$ of the trial $(\mathrm{CT} 0-\mathrm{CT} 3, \mathbf{e})$ or $(\mathrm{CT} 12-\mathrm{CT} 15, \mathbf{g})$ for $m \mathrm{Wake}^{(+/+)}(\mathrm{n}=8$, grey)

911 vs $m W_{a k e}^{(--)}(\mathrm{n}=8$, red) mice. Data were collected from 3 independent trials. $\mathbf{f}$ and $\mathbf{h}$,

912 Habituation of animals shown in (e) and (g), respectively, as assessed by total distance

913 (cm) traveled in the first 30 mins vs the last 30 mins of the trial. i, Locomotor activity

914 profile over $24 \mathrm{hrs}$ for $m W_{a k e}^{(\mathrm{Cre} /+)}\left(\mathrm{n}=9\right.$, grey) and $m W_{a k e}^{(\mathrm{Cre} / \mathrm{Cre})}(\mathrm{n}=10$, red) under DD

915 conditions. $\mathbf{j}$, Total locomotor activity (total number of beams broken along $\mathrm{X}$ and $\mathrm{Y}$

916 axes) from the mice in (i) at CT0-12 vs CT12-24.

918 Extended Data Figure 3 | mWAKE inhibits the activity of SCN neurons at night

919 a, Schematic of whole-cell patch-clamp recordings from $\mathrm{mWake}^{+}$neurons (tdTomato ${ }^{+}$

920 neurons in $\mathrm{mWake} e^{(\mathrm{Cre} /+)}$ or $\left.\mathrm{mWake}{ }^{(\mathrm{Cre} / \mathrm{Cre})} \mathrm{mice}\right)$, in the core region of the SCN (top).

921 Representative images of a biocytin- and tdTomato-labeled cell post-recording (bottom). 
922 Scale bar denotes $25 \mu \mathrm{m}$. b and c, $f-I$ curves for $m W a k e^{+} \mathrm{SCN}$ neurons from $m$ Wake $e^{(\mathrm{Cre} /+)}$

923 vs $m$ Wake ${ }^{(\text {Cre/Cre) }}$ mice at ZT0-2 (n=21 and 19) (b) or ZT12-14 (n=19 and 13) (c). d,

924 Representative traces of voltage-clamp recordings of $m W_{a k e}^{+} \mathrm{SCN}$ neurons of

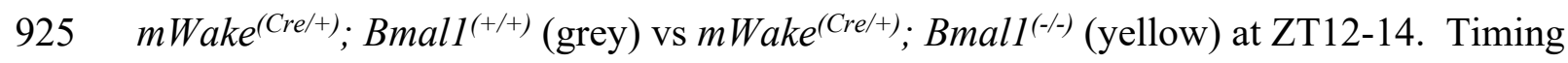

926 of GABA $(1 \mathrm{mM})$ application is shown. e, GABA-evoked current in $m W a k e^{S C N}$ neurons

927 from $\mathrm{mWake}^{\left(\mathrm{Cre} /+^{+}\right)}$; Bmal1 ${ }^{(++)}(\mathrm{n}=24)$ or Wake $^{(\mathrm{Cre} /+)}$; Bmal1 ${ }^{(-/)}(\mathrm{n}=26)$ mice at ZT12-14.

$928 \mathrm{n}$ represents individual cells from 4 animals.

930 Extended Data Figure 4 | Loss of mWAKE in SCN does not cause hyperactivity

931 a, Schematic of the genomic structure of the $m$ Wake locus and insertion of lox $P$ sites

932 flanking exons 4 and 5 in the $m W a k e^{(f l o x)}$ allele. b and d, Locomotor activity profile over

$93324 \mathrm{hrs}$ for $m W a k e^{(f l o x /-)}\left(\mathrm{n}=5\right.$, grey) and $\operatorname{Six}^{(\mathrm{Cre} /+)} ; m W a k e^{(f l o x /-)}(\mathrm{n}=6$, green) under LD (b)

934 or DD (d) conditions. c and e, Total locomotor activity from the mice in (b) at ZT0-12

935 and ZT12-24 or (d) at CT0-12 and CT12-24.

937 Extended Data Figure $5 \mid \mathbf{m} \mathrm{Wake}^{+}$DMH neurons promote arousal

938 a, Schematic of whole-cell patch-clamp recordings from $\mathrm{mWake}^{+}$neurons (tdTomato ${ }^{+}$

939 neurons in $m W a k e^{(\mathrm{Cre} /+)}$ or $\left.m W a k e^{(\mathrm{Cre} / \mathrm{Cre})} \mathrm{mice}\right)$, in the DMH region (top). Representative

940 images of a biocytin- and tdTomato-labeled cell post-recording (bottom). Scale bar

941 denotes $25 \mu \mathrm{m}$. b and c, $f$-I curves for $m W a k e^{D M H}$ neurons from $m W$ ake $^{\left(\mathrm{Cre}^{++}\right)} \mathrm{vs}$

942 mWake $^{(\text {Cre/Cre) }}$ mice at ZT0-2 (n=11 and 11, Day) (b) or ZT12-14 (n=8 and 8, Night) (c).

$943 \mathrm{n}$ represent individual cells from 4 animals. d and e, NREM (d) and REM sleep (e) (\%

944 per hr bin) for $m W a k e^{(\mathrm{Cre} /+)}$ mice with AAV-DIO-DREADD-Gq injected bilaterally into

945 the DMH, following IP injection of vehicle (grey) or $1 \mathrm{mg} / \mathrm{kg} \mathrm{CNO}$ (magenta) at ZT6.

946 Note these are data are from the same mice as in Fig. 21. f, Total locomotor activity (total

947 number of beams broken along $\mathrm{X}$ and $\mathrm{Y}$ axes) of $m W a k e^{(\mathrm{Cre} /+)}$ mice with bilateral

948 injections of AAV-DIO-DREADD-Gi into the DMH in the $4 \mathrm{hrs}$ following IP injection

949 of vehicle (grey) vs CNO (1 mg/kg, magenta) $(\mathrm{n}=4)$ at ZT10. g, Representative short-

950 time Fourier transform spectrograms of 4 hrs of recorded EEG activity, starting after IP

951 injection of vehicle alone (above) or $3 \mathrm{mg} / \mathrm{kg} \mathrm{CNO}$ (below) at ZT10, from $\mathrm{mWake} e^{(\mathrm{Cre} /+)}$

952 mice injected with AAV-DIO-DREADD-Gi bilaterally into the DMH. h-j, Wakefulness

953 (h), NREM (i), and REM (j) amount for the mice described in (g), plotted as \% time in 1 
$954 \mathrm{hr}$ bins following IP injection of vehicle (grey) or $3 \mathrm{mg} / \mathrm{kg} \mathrm{CNO}$ (magenta) (n=4) at

955 ZT10. $\mathbf{k}$, total locomotor activity (total number of beams broken along X and Y axes) of

$956 m W a k e^{\left(\mathrm{Cre}^{+}\right)}$mice with sham injections into the DMH in the 4 hrs following IP injection

957 of vehicle (grey), CNO (1 mg/kg, cyan), or CNO (3 mg/kg, magenta) (n=3) at ZT6. I,

958 Vigilance state (\%) of 2 hrs of recorded EEG activity, starting after IP injection of vehicle

959 alone, $1 \mathrm{mg} / \mathrm{kg} \mathrm{CNO}$, or $3 \mathrm{mg} / \mathrm{kg} \mathrm{CNO}$ at ZT6, from the mice described in (k).

961 Extended Data Figure 6 | Additional data related to single cell RNA sequencing

962 a, UMAP plot showing distribution of $\mathrm{mWake}^{+}$cells in individual scRNA-Seq libraries

963 and distribution of $m W_{a k e}^{+}$neurons and ependymal cells. b, Violin plot showing

964 distribution of number and mean (black dot) of genes (top) and total mRNAs (bottom,

965 calculated by the number of unique molecular identifiers (UMIs)) in individual scRNA-

966 Seq libraries. c, Heatmap showing key marker genes that were used to identify spatial

967 location of each $m W_{a k e}^{+}$neuronal cluster. d-g, Representative images of FISH

968 experiments using RNAscope probes against tdTomato, Vglut2, and/or Vgat mRNA in

969 the DMH (d and e) and SCN (f and $\mathbf{g}$ ) of $m$ Wake $e^{(\mathrm{Cre} /+)}$ mice. Nuclei counterstained with

970 DAPI. Scale bars denote $200 \mu \mathrm{m}$ in $\mathbf{d}$ and $\mathbf{f}$ and $20 \mu \mathrm{m}$ in e and $\mathbf{g}$.

972 Extended Data Figure 7 | Additional data related to neuromodulatory input to 973 mWake $\boldsymbol{e}^{\text {DMH }}$ neurons

974 a, Schematic showing dual injections of the Cre-dependent rabies helper virus (AAV-

975 FLEX-G) and the rabies- $\Delta \mathrm{G}-\mathrm{BFP}(\mathrm{RabV}-\Delta \mathrm{G}-\mathrm{BFP})$ virus into the DMH of $m$ Wake $e^{(\mathrm{Cre} /+)}$

976 mice. b-e, Representative images of BFP fluorescence and anti-histamine decarboxylase

977 (HDC) (b), anti-Orexin A (OxA) (c), anti-choline acetyltransferase (CHAT) (d), or anti-

978 tyrosine hydroxylase (TH) (e), as well as merged images from TMN (b), lateral

979 hypothalamus (LH) (c), BF (d), or LC (e) regions, from the mice described in (a). Scale

980 bar denotes $20 \mu \mathrm{m}$ in (b), $50 \mu \mathrm{m}$ in (c), $20 \mu \mathrm{m}$ in (d), $20 \mu \mathrm{m}$ in (e). f, Schematic showing

981 patch-clamp recordings of $m W a k e^{D M H}$ neurons from $m W_{a k e}^{(\mathrm{Cre} /+)}$ mice, following

982 application of histamine. $\mathbf{g}$, Representative membrane potential traces from the

983 recordings described in (f) in the presence of synaptic blockers at ZT12-14. $\mathbf{h}$,

984 Spontaneous mean firing rate of $m W a k e^{D M H}$ neurons following application of $20 \mu \mathrm{M}$ 
985 histamine (n=8 and 9 cells) at ZT12-14 in the absence ("- blockers") or presence ("+

986 blockers") of $20 \mu \mathrm{M}$ CNQX, $50 \mu \mathrm{M}$ AP5, and $10 \mu \mathrm{M}$ picrotoxin. The neurons recorded

987 from in (h) were derived from 3 animals. i, Schematic showing unilateral stereotaxic

988 injection of AAV-viral vector expressing Cre-dependent eGFP (AAV-FLEX-eGFP) into

989 the DMH of $m$ Wake $e^{(\mathrm{Cre} /+)}$ mice. j, Representative sagittal section showing broad

990 projection of GFP fluorescence following injection of AAV-FLEX-eGFP shown in (e).

991 Dashed ellipse denotes injection site, and locations of the basal forebrain (BF), cortex

992 (Cx), hypothalamus (Hyp), locus coeruleus (LC), and pons are noted. Scale bar denotes 1

$993 \mathrm{~mm}$.

995 Extended Data Figure 8 | The mWAKE network is critical for arousal

996 a, Representative images of anti-HA labeling of cells and processes expressing LSL-

997 DREADD-Gi, tdTomato fluorescence from $\mathrm{mWake}^{+}$cells, or merged images in

998 representative brain regions from $m$ Wake $e^{(\mathrm{Cre} /+)} ; L S L-D R E A D D-G i$ mice. Sections

999 containing suprachiasmatic nucleus (SCN), basal forebrain (BF), and cortex $(\mathrm{Cx})$ are

1000 shown. Scale bar denotes $100 \mu \mathrm{m}$ for SCN, $20 \mu \mathrm{m}$ for BF, and $100 \mu \mathrm{m}$ for Cx panels.

1001 b,c, Two additional short-time Fourier transform spectrograms of 12 hrs of recorded EEG

1002 activity from $m W a k e^{\left(\mathrm{Cre}^{+}\right)}$; $L S L-G i$ mice after IP injection at ZT10 of vehicle alone

1003 (above) or $0.3 \mathrm{mg} / \mathrm{kg} \mathrm{CNO}$ (below). These panels illustrate the range of EEG phenotypes

1004 observed. d, Jitter plot showing behavioural classification of arousal level for $H D C^{(\mathrm{Cre} /+)}$;

$1005 L S L-G i$ mice following injection of vehicle or $1 \mathrm{mg} / \mathrm{kg} \mathrm{CNO}(\mathrm{n}=4)$. e, Representative

1006 short-time Fourier transform spectrograms of $12 \mathrm{hrs}$ of recorded EEG activity from

$1007 H D C^{\left(C^{(-)}\right)} ;$LSL-Gi mice after IP injection at ZT10 of vehicle alone (above) or $1 \mathrm{mg} / \mathrm{kg}$

1008 CNO (below). f, Short-time Fourier transform spectrograms for a $H D C^{\left(\mathrm{Cre}^{++}\right)} ; L S L-G i$

1009 mouse starting 150 mins after injection of vehicle (left) or $1 \mathrm{mg} / \mathrm{kg} \mathrm{CNO}$ (right) at ZT10.

1010 The dashed red box indicates the time window used for spectral and amplitude analysis in

1011 (g) and (h). g, Welch's power spectral density estimates as a percentage of total EEG

1012 power across $10 \mathrm{~min}$, averaged across multiple EEG traces. $H D C^{\left(\mathrm{Cre}^{+}\right)} ; L S L-G i$ injected

1013 with vehicle (grey) or $1 \mathrm{mg} / \mathrm{kg}$ CNO (green) $(\mathrm{n}=3)$. Inset shows a plot of delta-band

1014 power as a percentage of total EEG power. h, EEG trace amplitude (plotted as

1015 normalized root mean square (RMS)) for the animals described in (f). 
1017 Supplementary Table 1 | Additional electrophysiological properties for $\boldsymbol{m} W a k e^{S C N}$

1018 and $\boldsymbol{m} W_{a k} e^{D M H}$ neurons.

1019

Supplementary Table 2 | Stereotaxic coordinates and viruses injected

Supplementary Table 3 | List of differentially expressed genes in $m \mathrm{Wake}^{+}$neurons

Supplementary Video 1 | mWake KO exhibit nighttime hyperactivity and circling.

$1025 \quad(\mathbf{0 : 0 0}-\mathbf{0 : 1 5})$, A single $m W a k e^{(-/)}$mouse exhibiting elevated homecage locomotor

1026 activity, compared to heterozygote littermates. (0:15- 0:19), Wake $^{(--)}$mouse

1027 demonstrating rapid $(\sim 1.6 \mathrm{~Hz})$ tight circling behavior. $(\mathbf{0 : 2 0}-\mathbf{0 : 3 1})$, Two

$1028 m W a k e^{(--)}$mice display broad circling activity $(\sim 1.4 \mathrm{~Hz})$. Video speed is not adjusted.

Supplementary Video $2 \mid m W a k e^{(--)}$mice are not impaired in the forced-swim assay. (0:00 - 0:40), Representative trial of forced swim assay for a control ("Wild Type") mouse. (0:40 - 1:22), Representative trial of forced swim assay for a $m W a k e^{(-/)}$ (“ $m$ Wake-KO”) mouse. (1:22 - 2:08), Representative trial of forced swim assay of $m W a k e^{(N M F 9 /-)}$ ("NMF9/mWake-KO") mouse. All animals were allowed to swim for $\geq 30$

1035 s before being removed.

Supplementary Video 3 | Chemogenetic inhibition of $\mathbf{m} \mathrm{Wake}^{+}$network induces

1038 profound reduction in arousal.

(0:00 - 0:35), WWake $^{(\mathrm{Cre} /+)} ; L S L-G i$ mouse ("Vehicle") 90 min after injection of vehicle solution, classified as "Normal," exhibiting normal spontaneous locomotion and exploration, as well as avoidance of paper object. (0:36 - 1:10), $\mathrm{mWake}^{(\mathrm{Cre} /+)} ; \mathrm{LSL}-\mathrm{Gi}$ mouse (“CNO\#1") 90 min after injection of $0.3 \mathrm{mg} / \mathrm{kg}$ CNO solution, classified as "Reduced Reactivity," displaying markedly decreased spontaneous locomotion, with

1044 intact righting reflex and reduced avoidance of paper object. (1:11 - 1:40),

$1045 m \mathrm{Wake}^{(\mathrm{Cre} /+)} ; \mathrm{LSL}-\mathrm{Gi}$ mouse (“CNO\#2”) $90 \mathrm{~min}$ after injection of $0.3 \mathrm{mg} / \mathrm{kg} \mathrm{CNO}$

1046 solution, classified as "Stuporous," with absent spontaneous locomotion, slow righting 1047 reflex, and minimal responsiveness to paper object. 
bioRxiv preprint doi: https://doi.org/10.1101/2020.03.12.989921; this version posted May 30, 2020. The copyright holder for this preprint (which was not certified by peer review) is the author/funder. All rights reserved. No reuse allowed without permission.

a

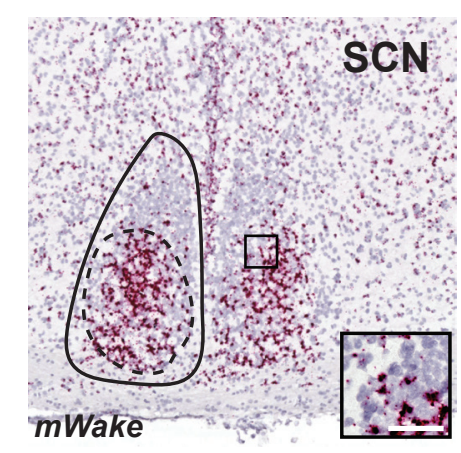

b

c
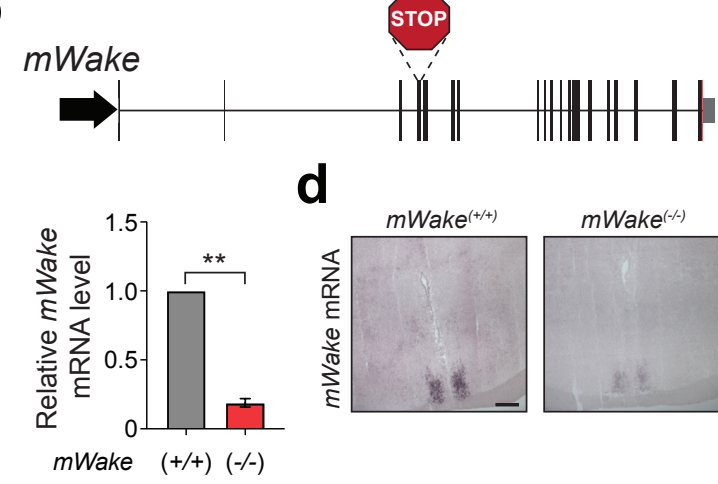

d

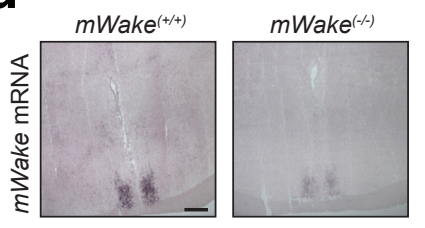

g

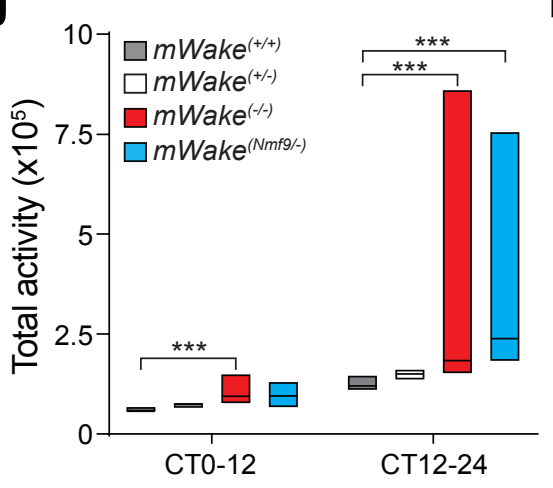

I

i
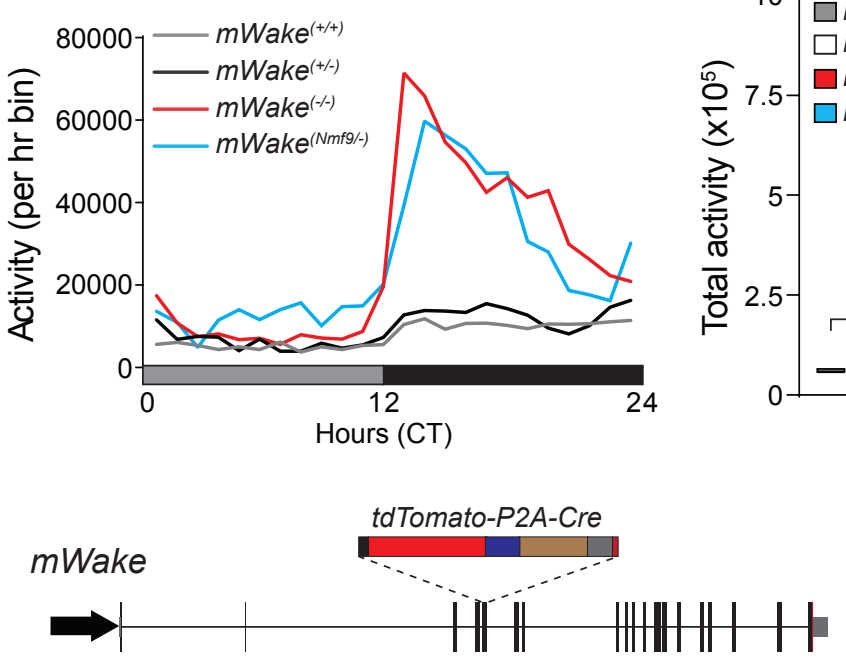

j

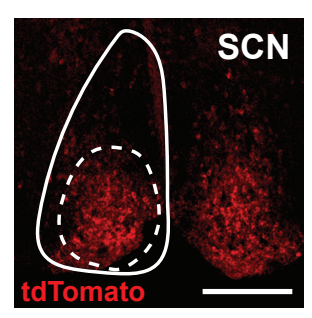

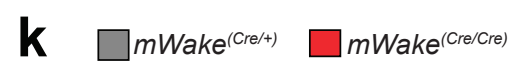

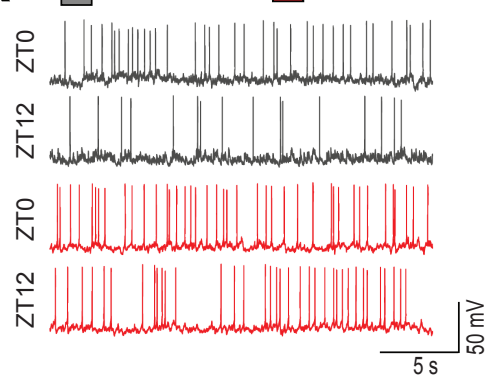

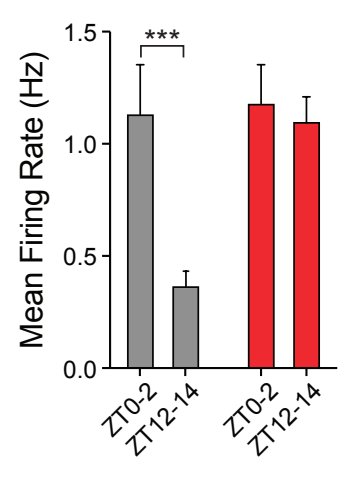

e

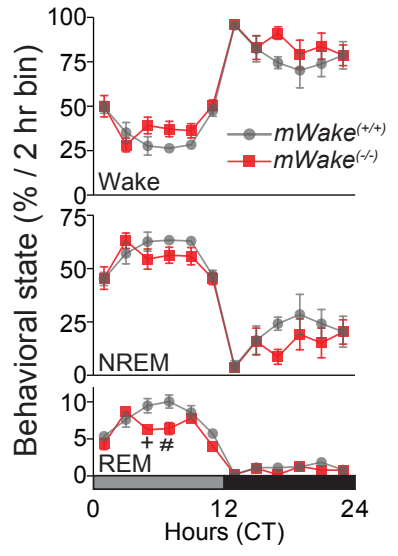

h

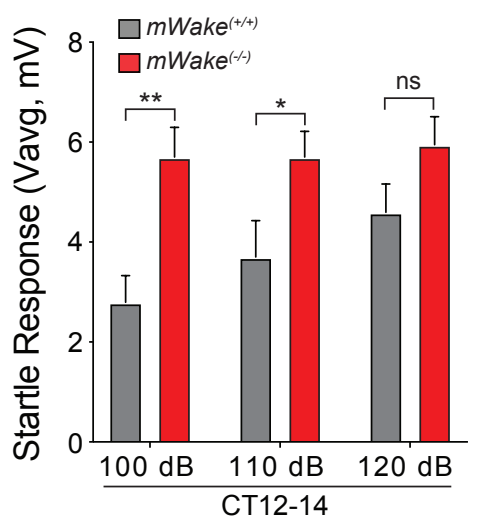

m

$\square m W a k e^{(\mathrm{Cre} /+)} \square m W a k e^{(\mathrm{Cr} / \mathrm{Cre})}$

n
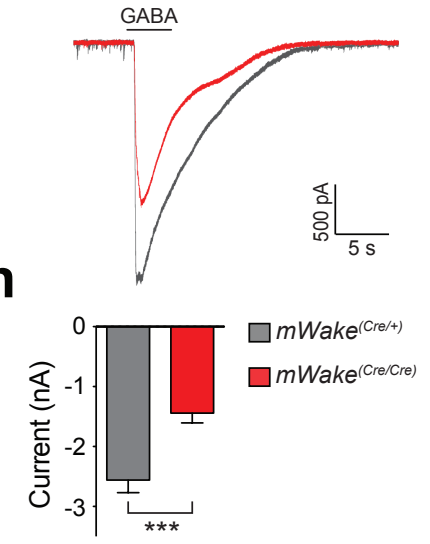
bioRxiv preprint doi: https://doi.org/10.1101/2020.03.12.989921; this version posted May 30, 2020. The copyright holder for this preprint (which

a

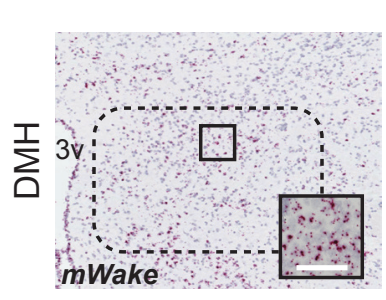

b

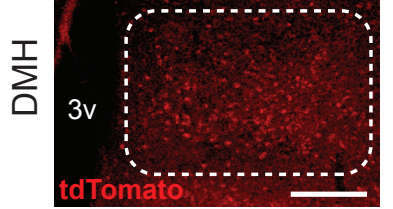

e

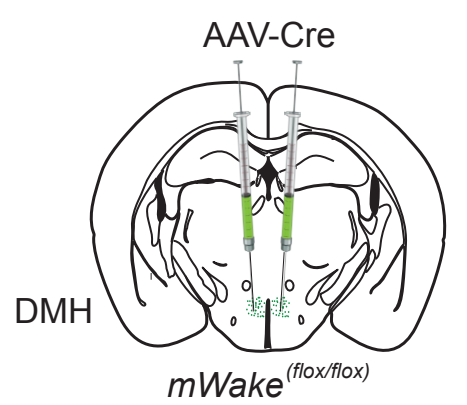

h

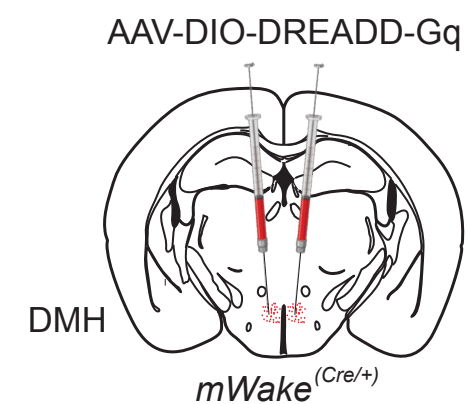

k

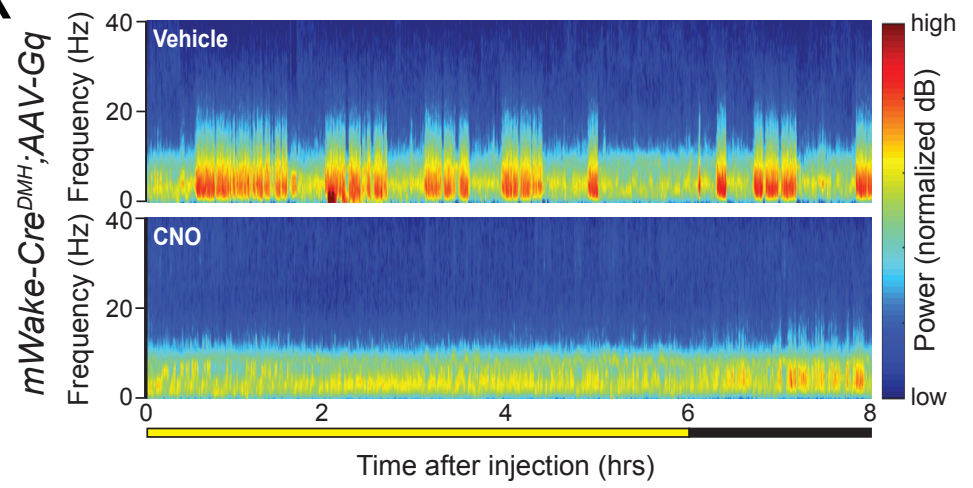

d

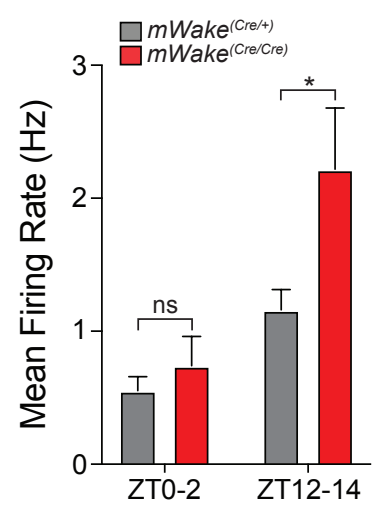

f

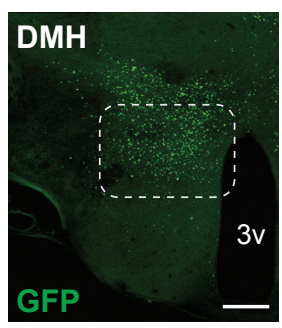

g

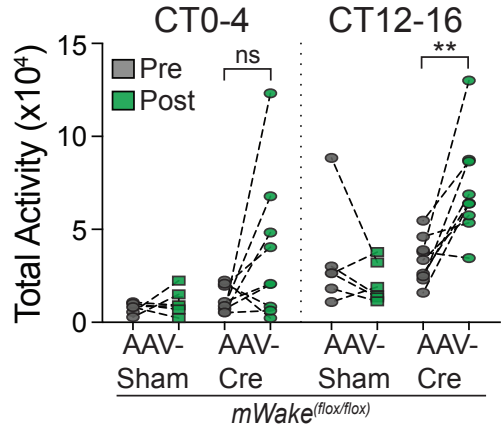

j

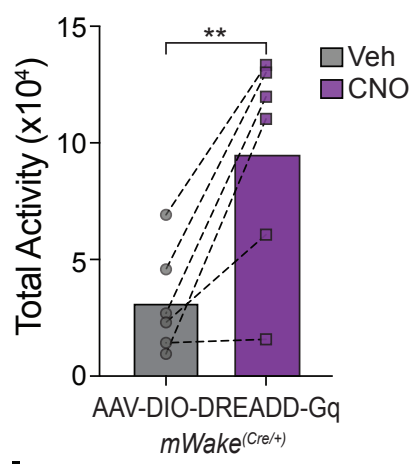


bioRxiv preprint doi: https://doi.org/10.1101/2020.03.12.989921; this version posted May 30, 2020. The copyright holder for this preprint (which

a mWake mYPOTHALAMUS $^{\text {Was not certified }}$

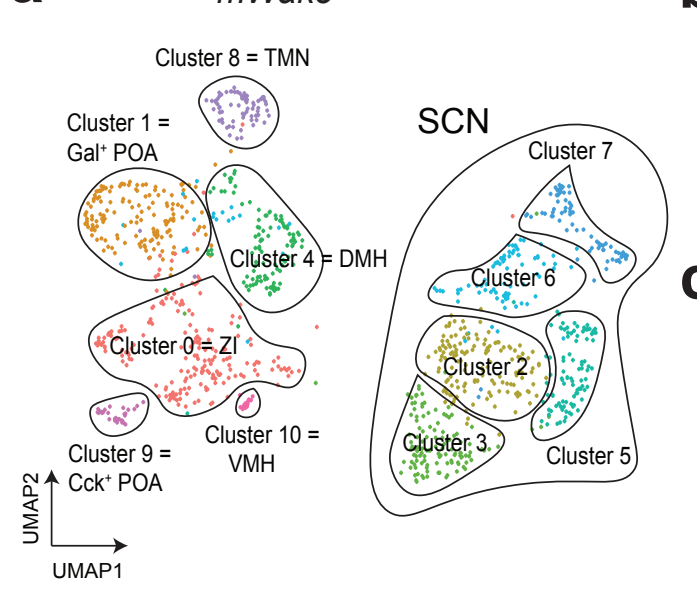

b $\square$ GABAergic $\square$ Glutamatergic $\square$ unknown

d

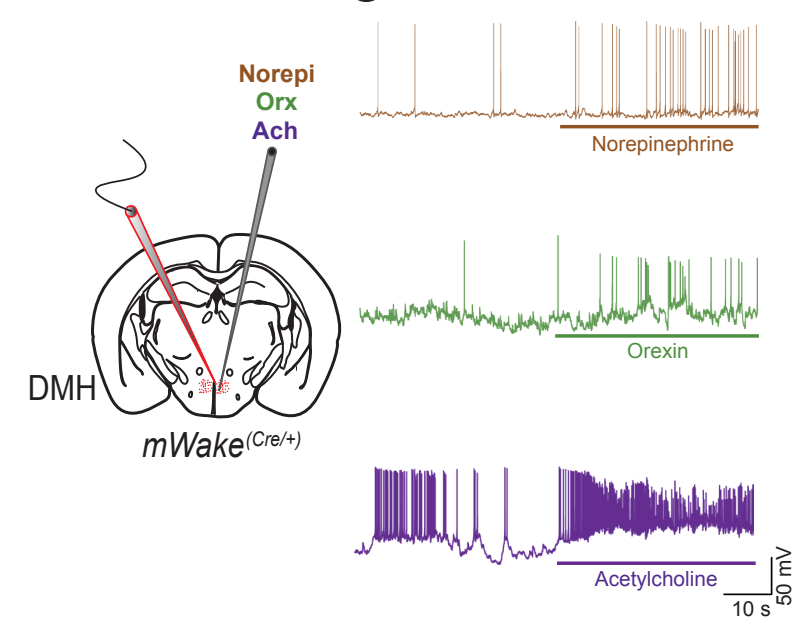

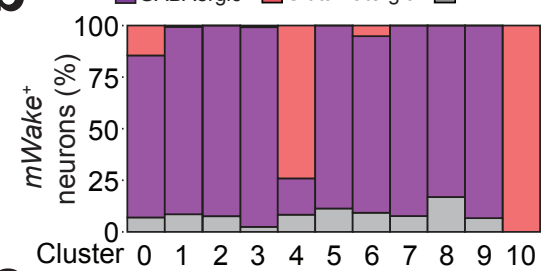

C
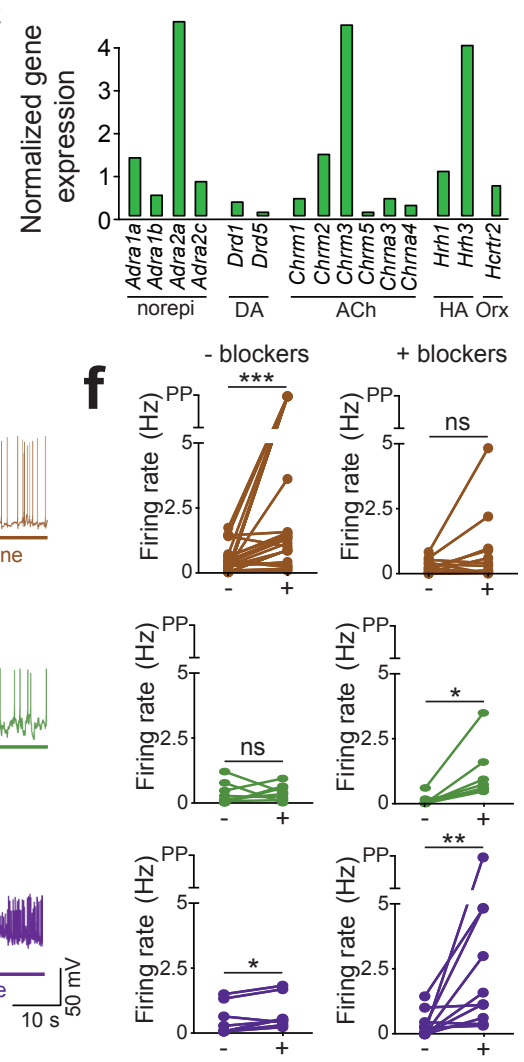

9
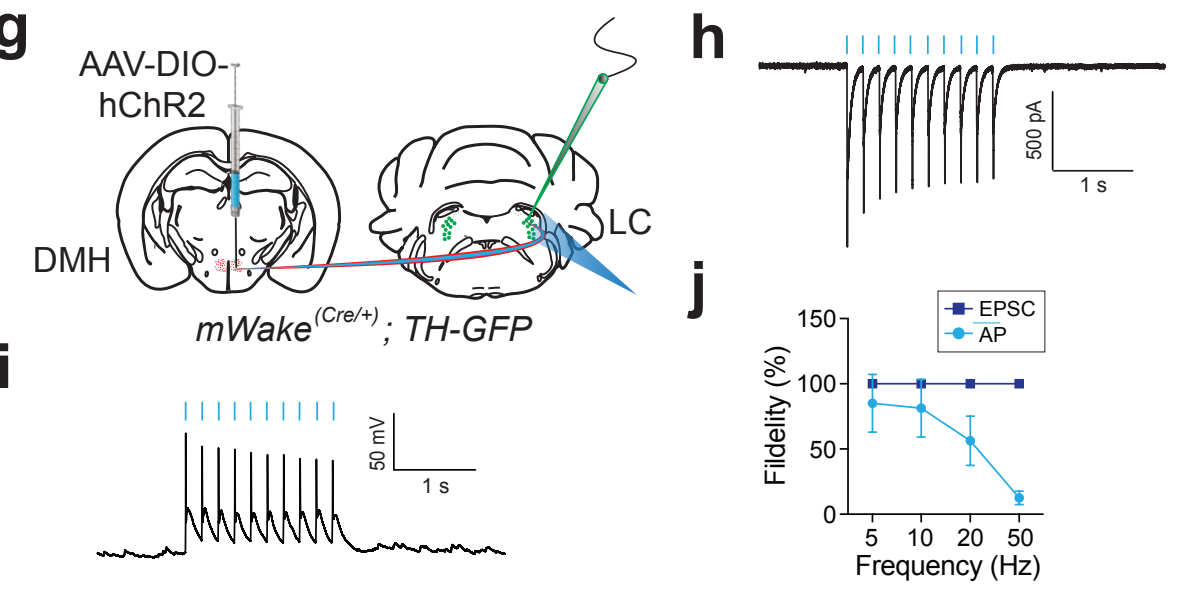
bioRxiv preprint doi: https://doi.org/10.1101/2020.03.12.989921; this version posted May 30, 2020. The copyright holder for this preprint (which was not certified by peer review) is the author/funder. All rights reserved. No reuse allowed without permission.

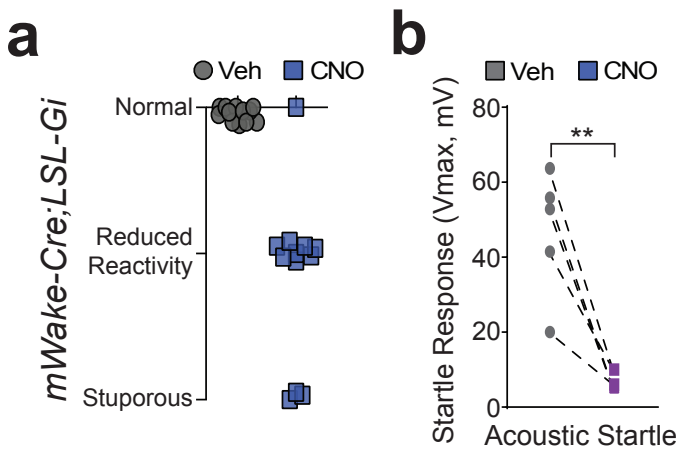

d
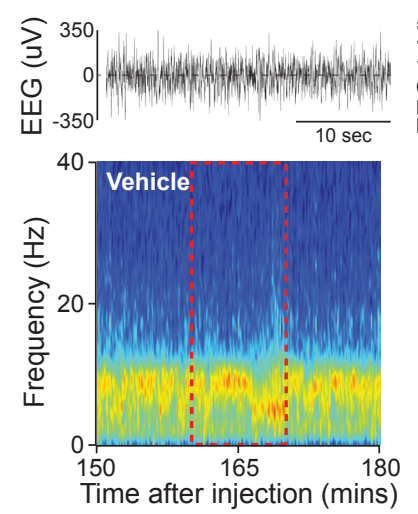
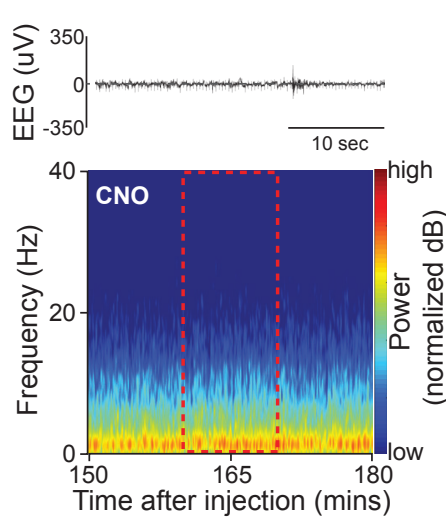

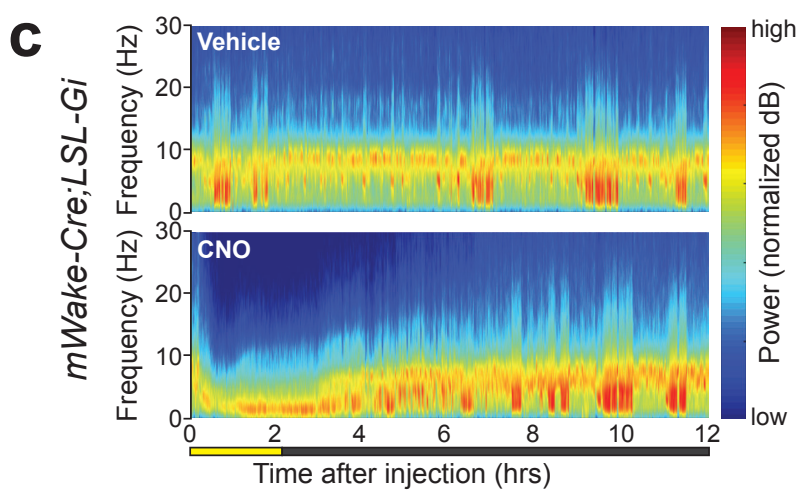

e

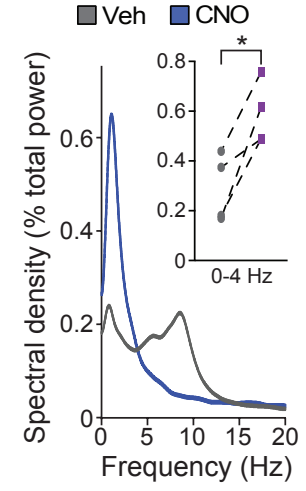

f

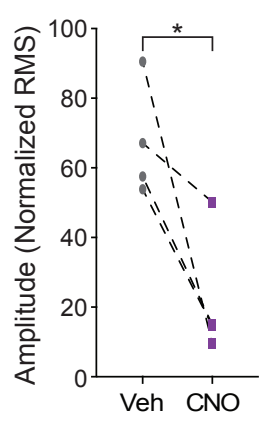


bioRxiv preprint doi: https://doi.org/10.1101/2020.03.12.989921; this version posted May 30, 2020. The copyright holder for this preprint (which was not certified by peer review) is the author/funder. All rights reserved. No reuse allowed without permission.

a

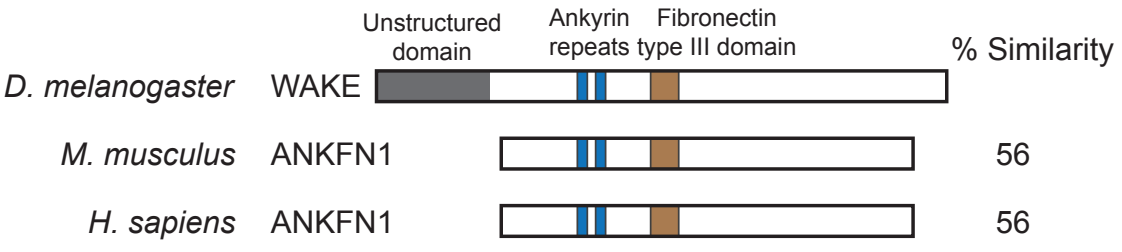

b

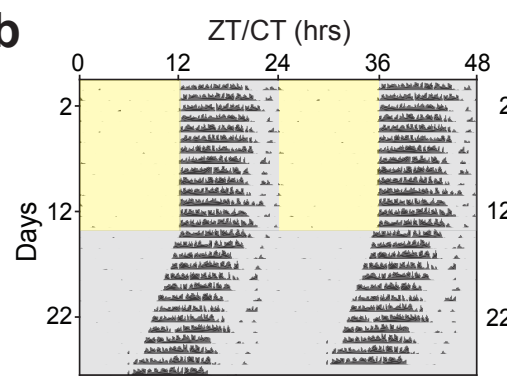

$m W a k e^{(+/+)}$

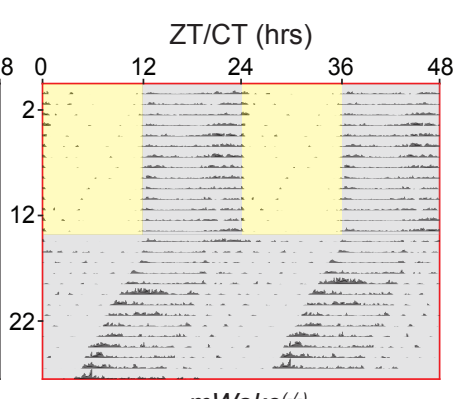

mWake(-/-)

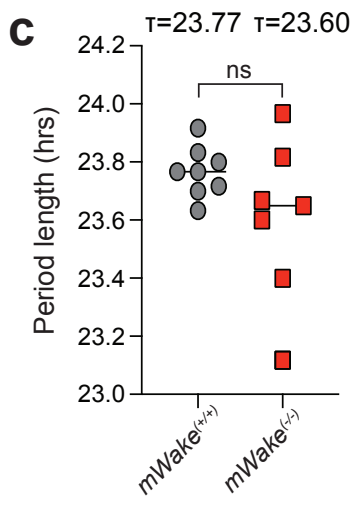

d
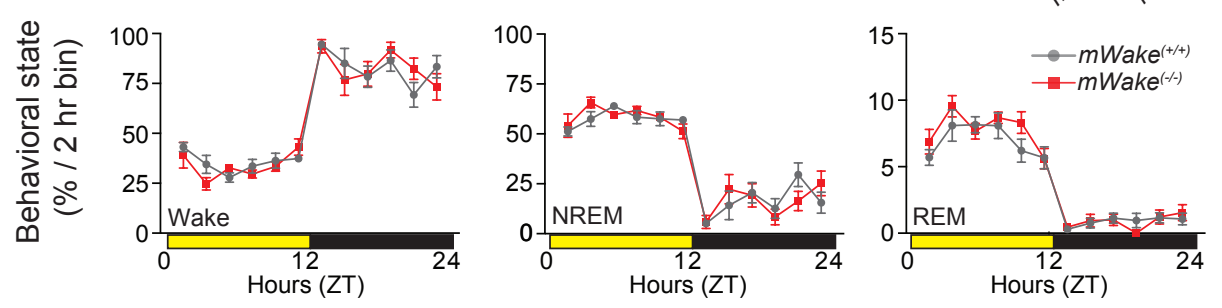

e
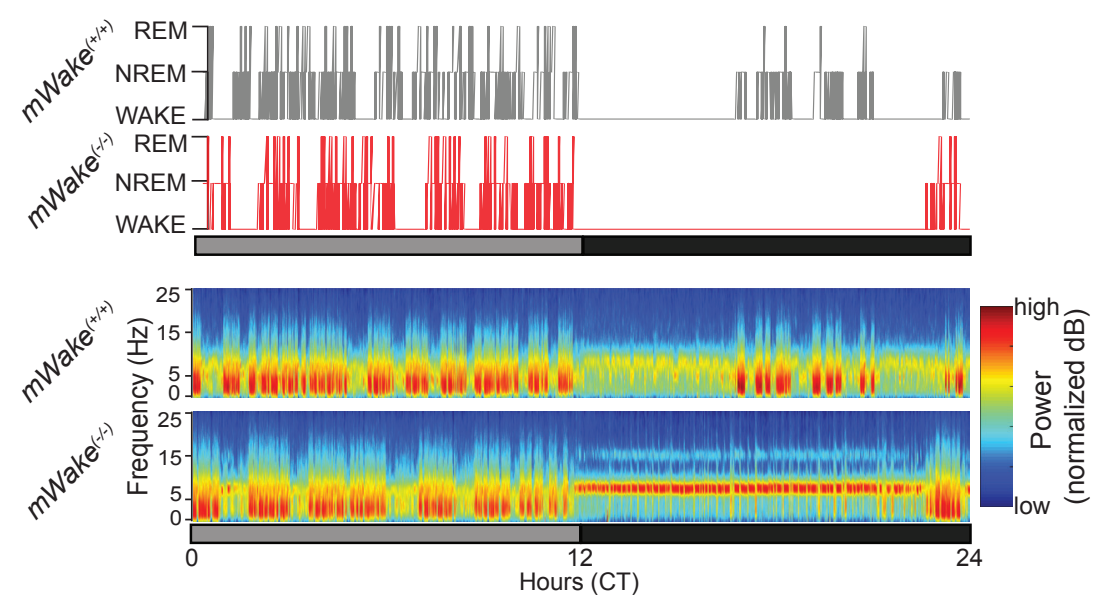

$\mathbf{f}$

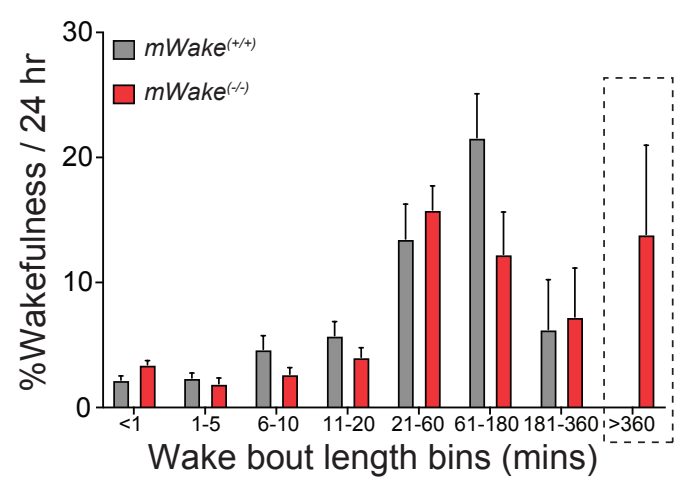


bioRxiv preprint doi: https://doi.org/10.1101/2020.03.12.989921; this version posted May 30, 2020. The copyright holder for this preprint (which

a

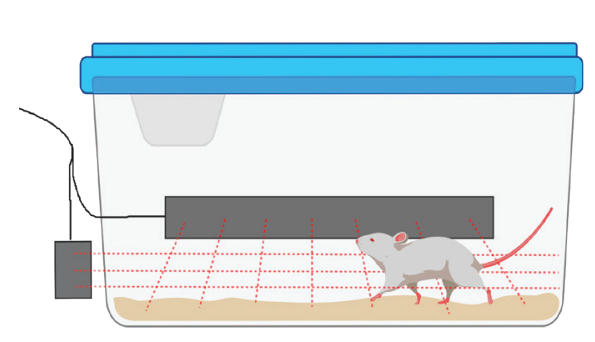

d

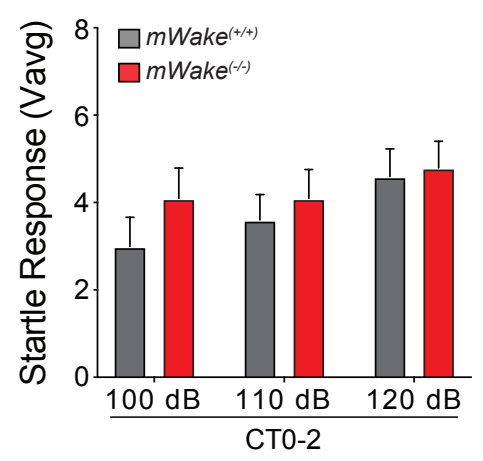

g

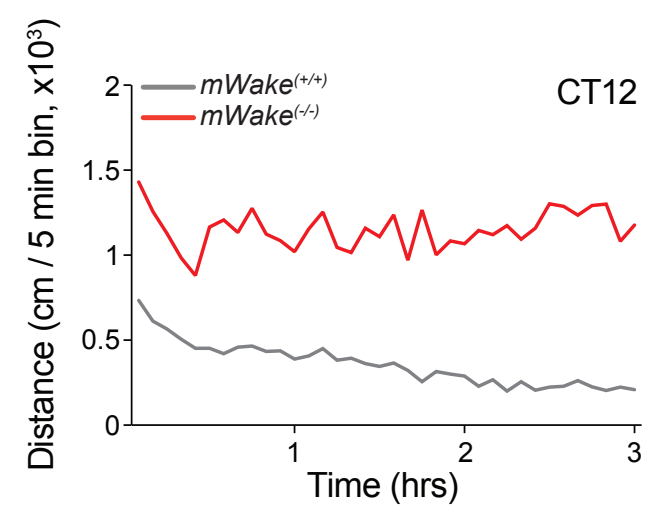

i

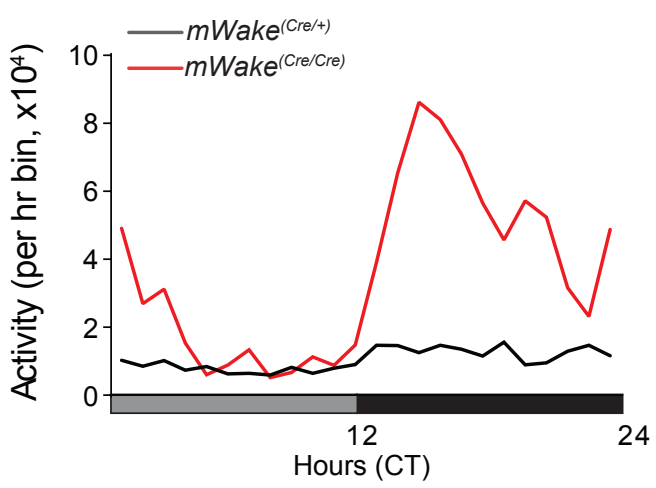

b

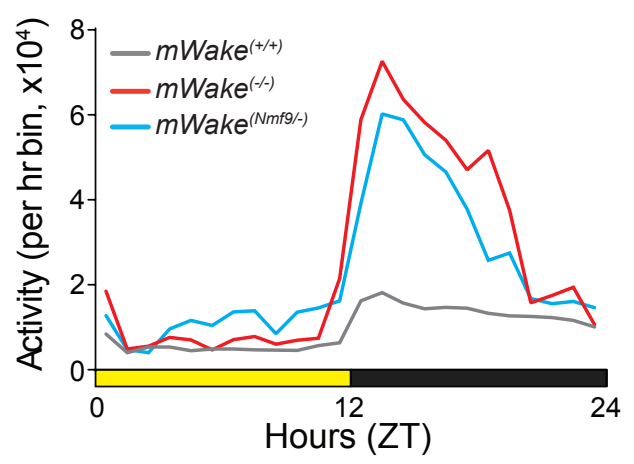

e

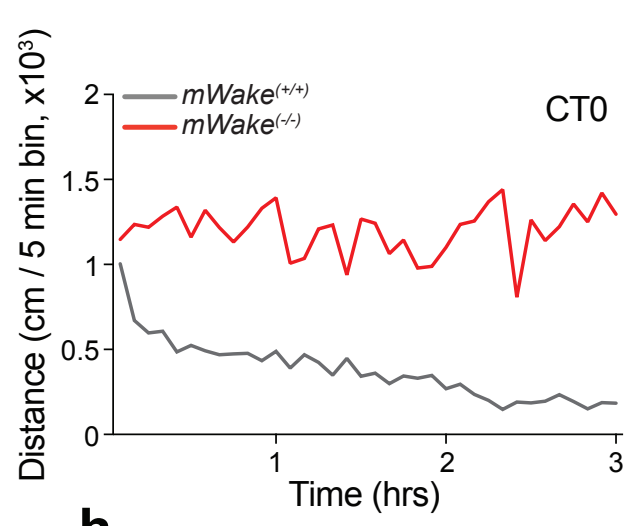

h
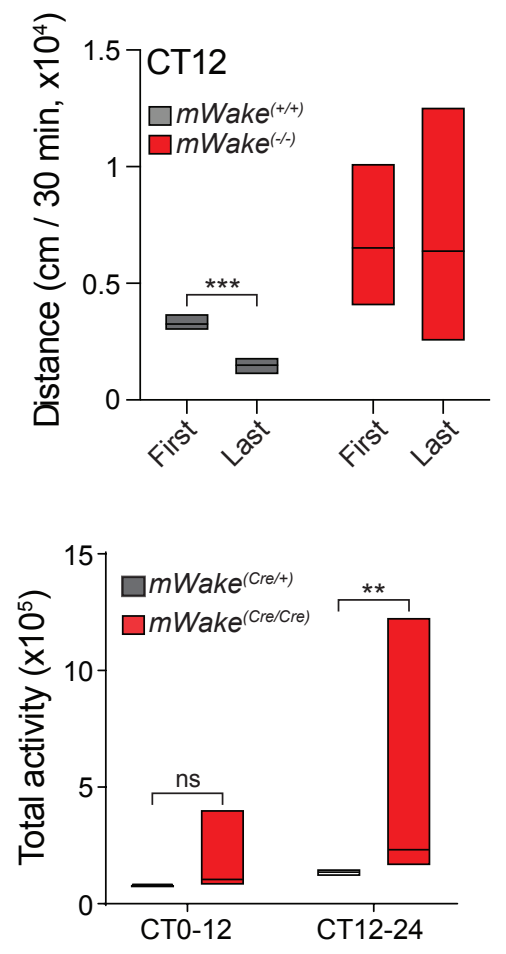

C

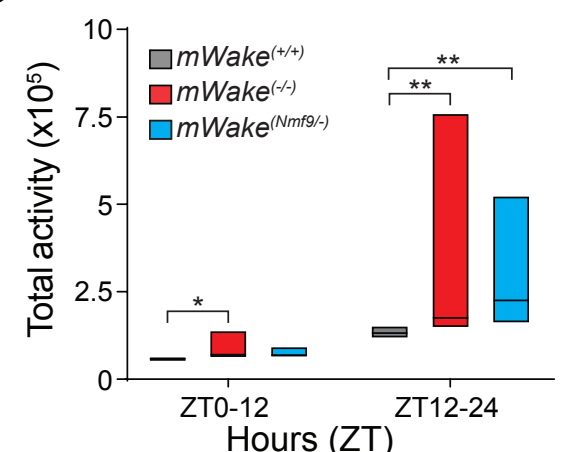

f

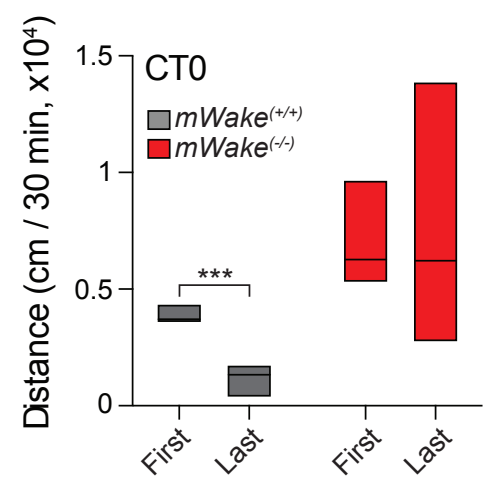


bioRxiv preprint doi: https://doi.org/10.1101/2020.03.12.989921; this version posted May 30, 2020. The copyright holder for this preprint (which was not certified by peer review) is the author/funder. All rights reserved. No reuse allowed without permission.

a

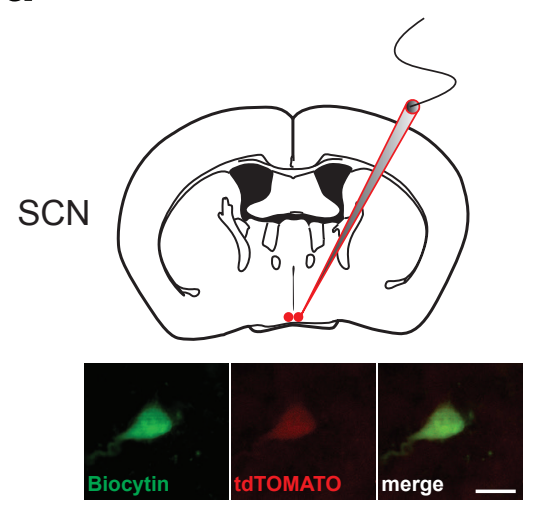

d

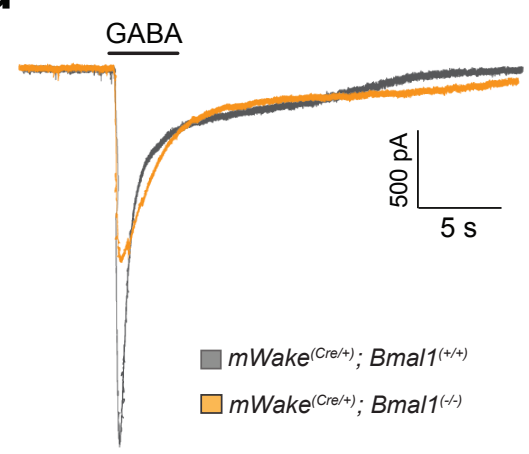

b

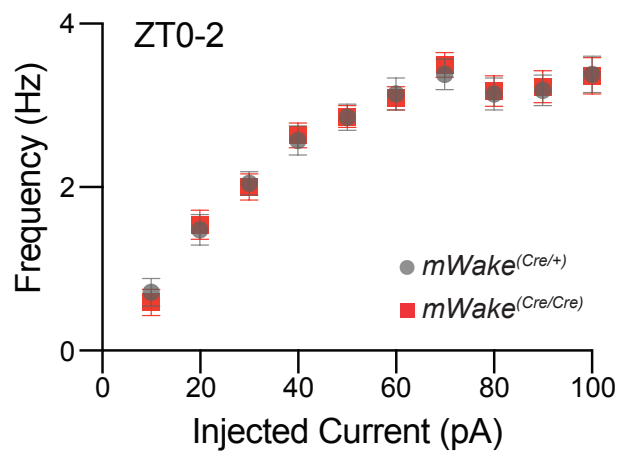

e

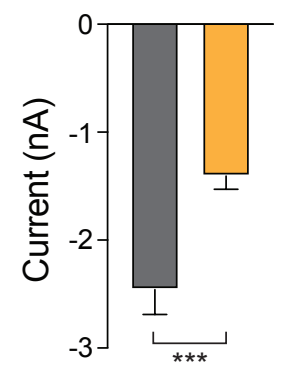

C

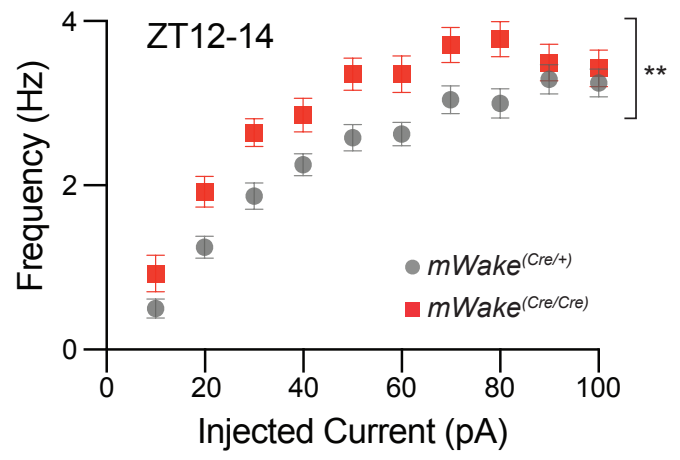


bioRxiv preprint doi: https://doi.org/10.1101/2020.03.12.989921; this version posted May 30, 2020. The copyright holder for this preprint (which was not certified by peer review) is the author/funder. All rights reserved. No reuse allowed without permission.

a

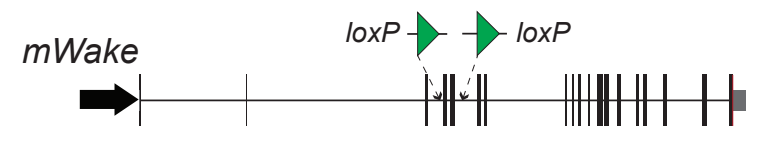

b

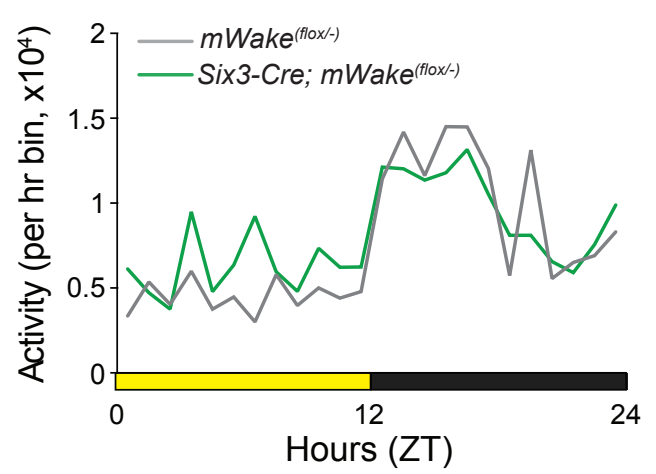

C

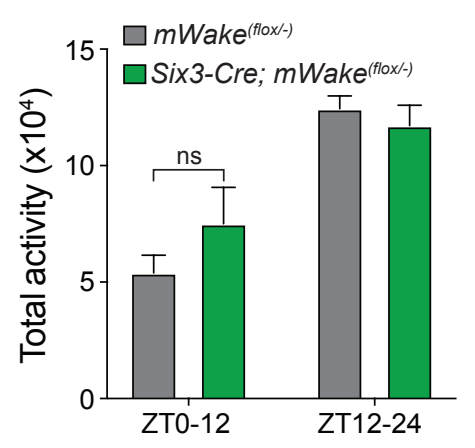

d

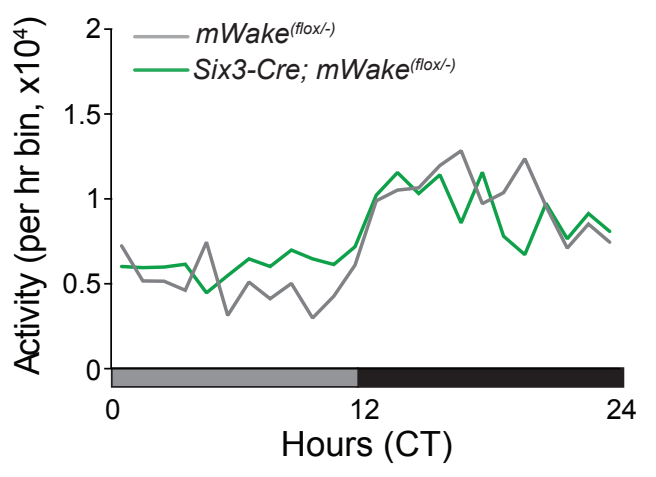

e

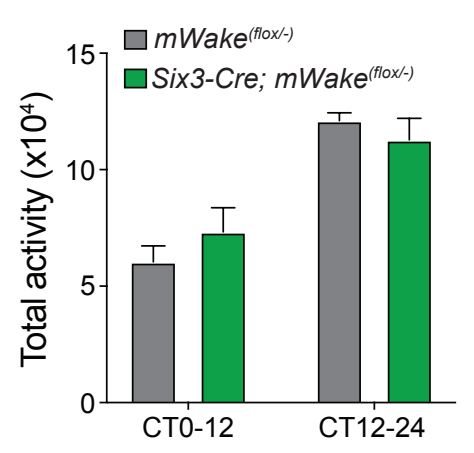


bioRxiv preprint doi: https://doi.org/10.1101/2020.03.12.989921; this version posted May 30, 2020. The copyright holder for this preprint (which was not certified by peer review) is the author/funder. All rights reserved. No reuse allowed without permission.

a

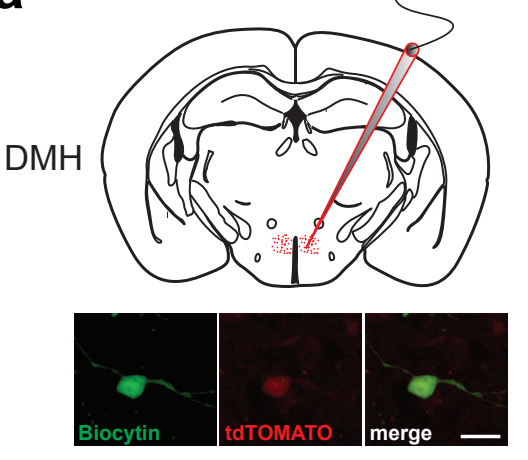

d

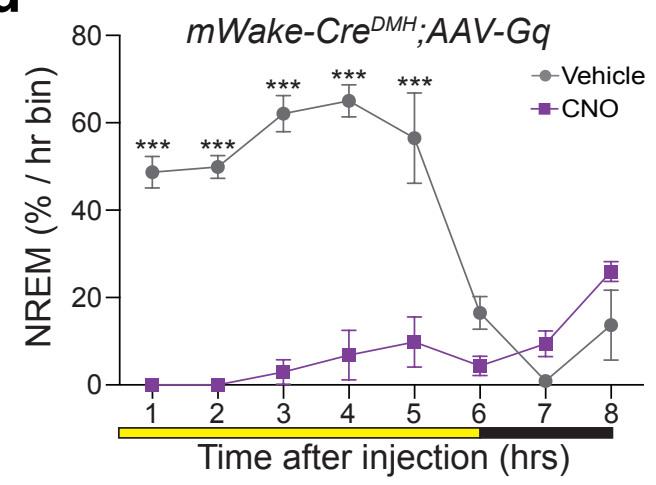

g

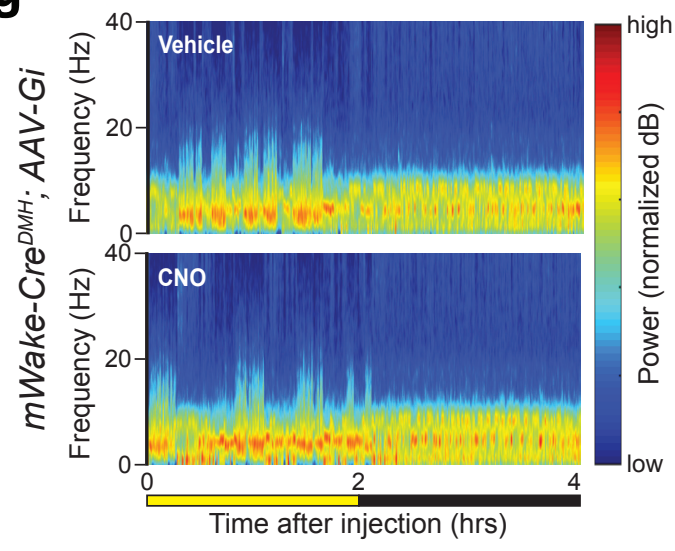

j b

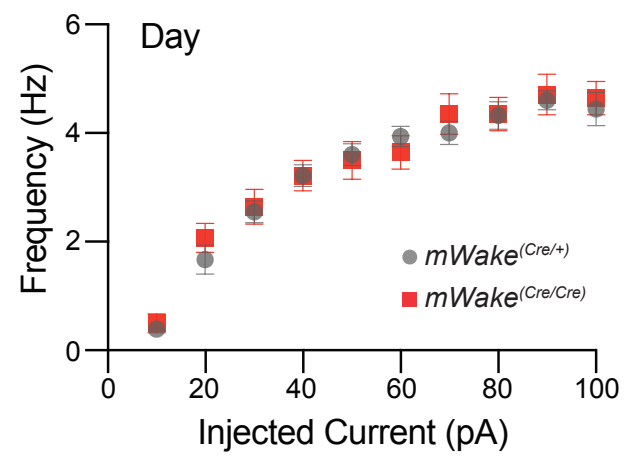

e

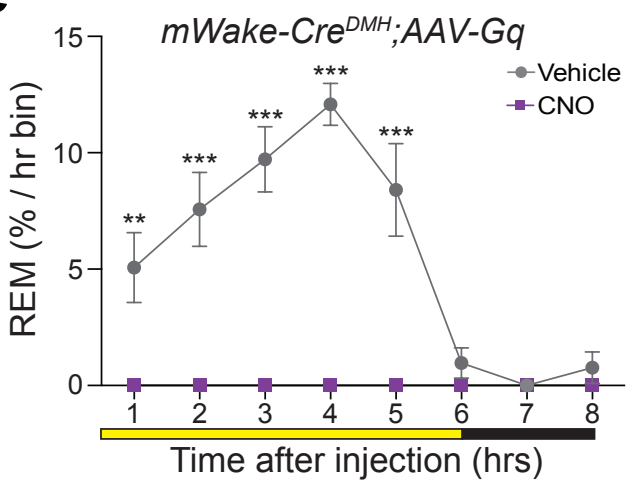

h

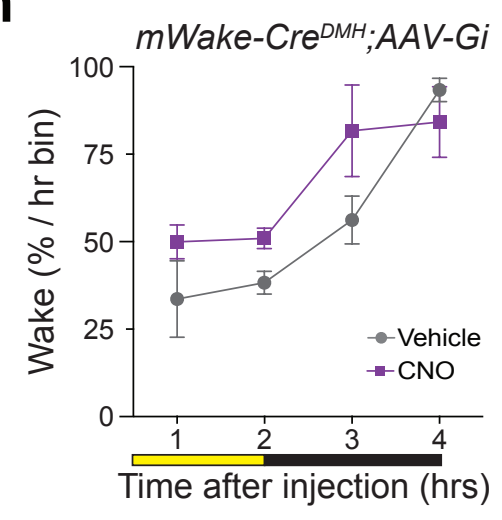

C

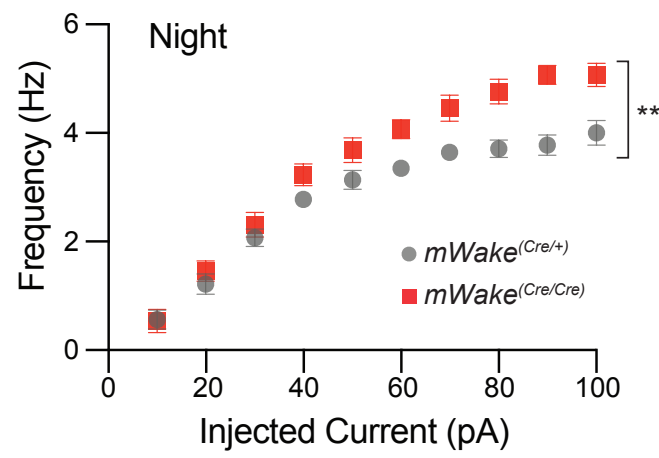

f
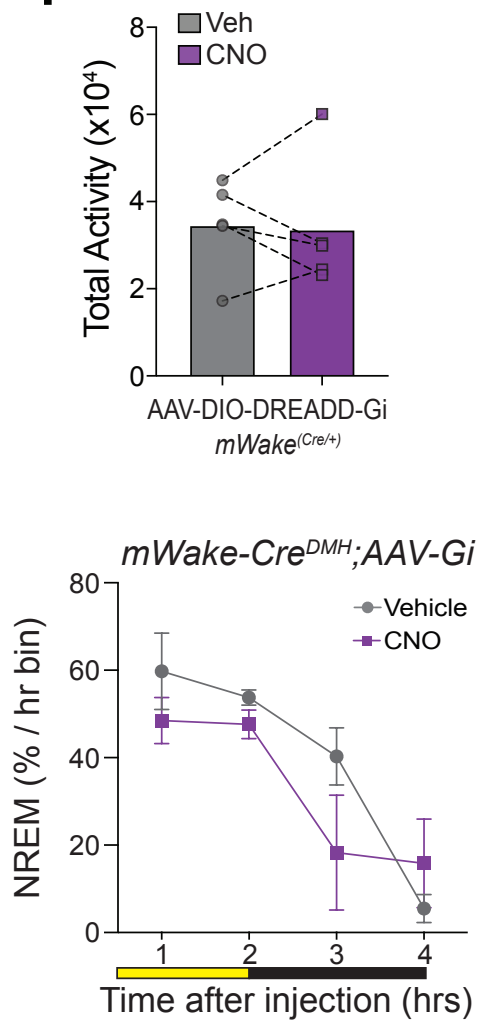
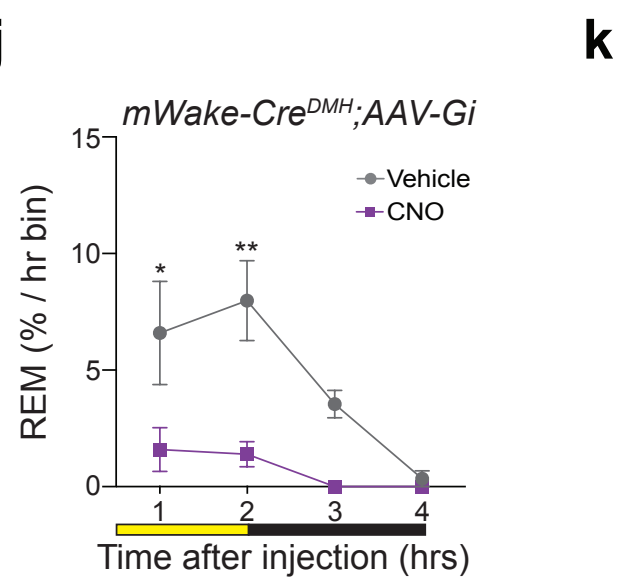

k

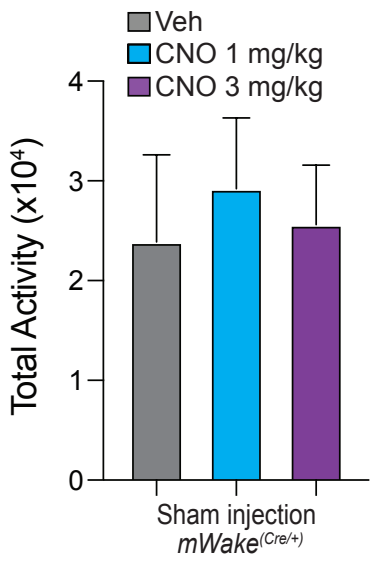

I

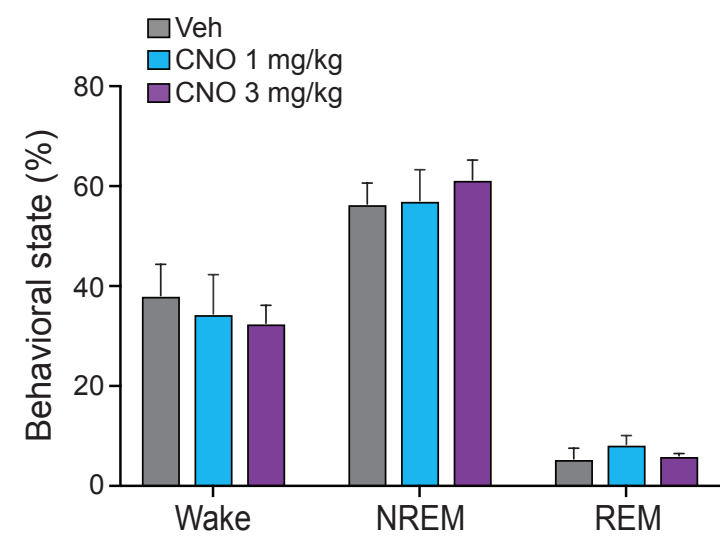


bioRxiv preprint doi: https://doi.org/10.1101/2020.03.12.989921; this version posted May 30, 2020. The copyright holder for this preprint (which was not certified by peer review) is the author/funder. All rights reserved. No reuse allowed without permission.

a

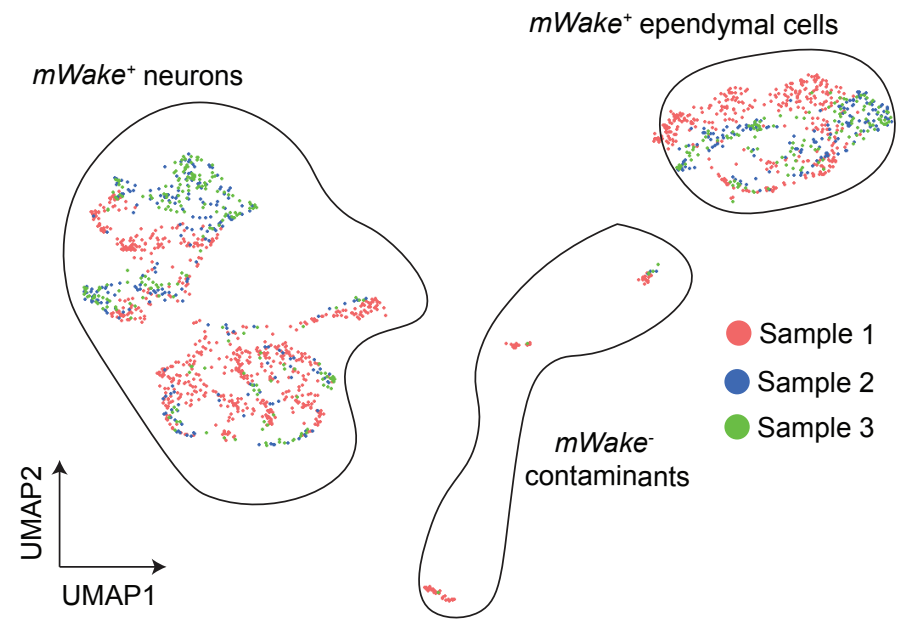

C

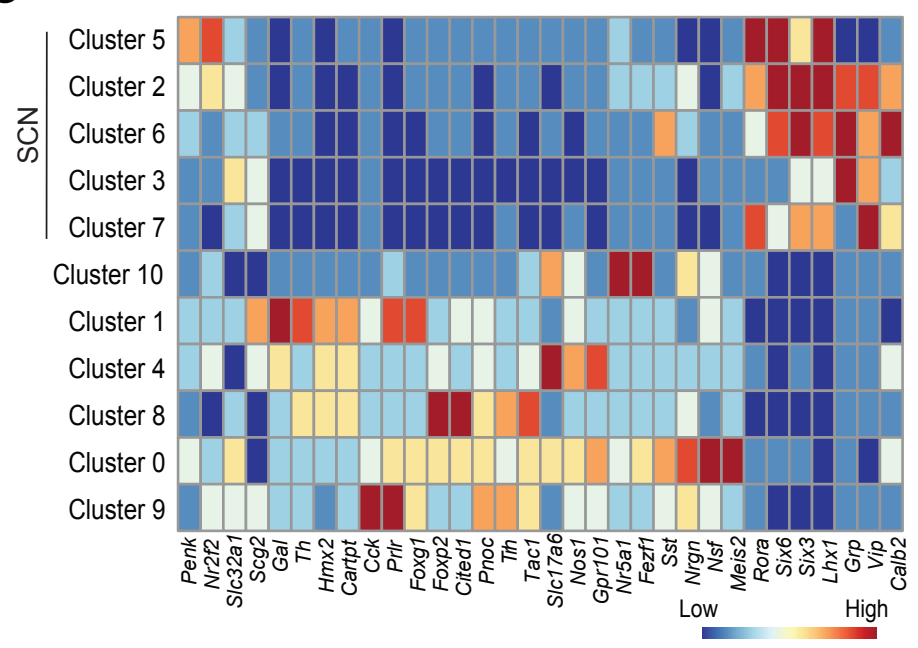

b
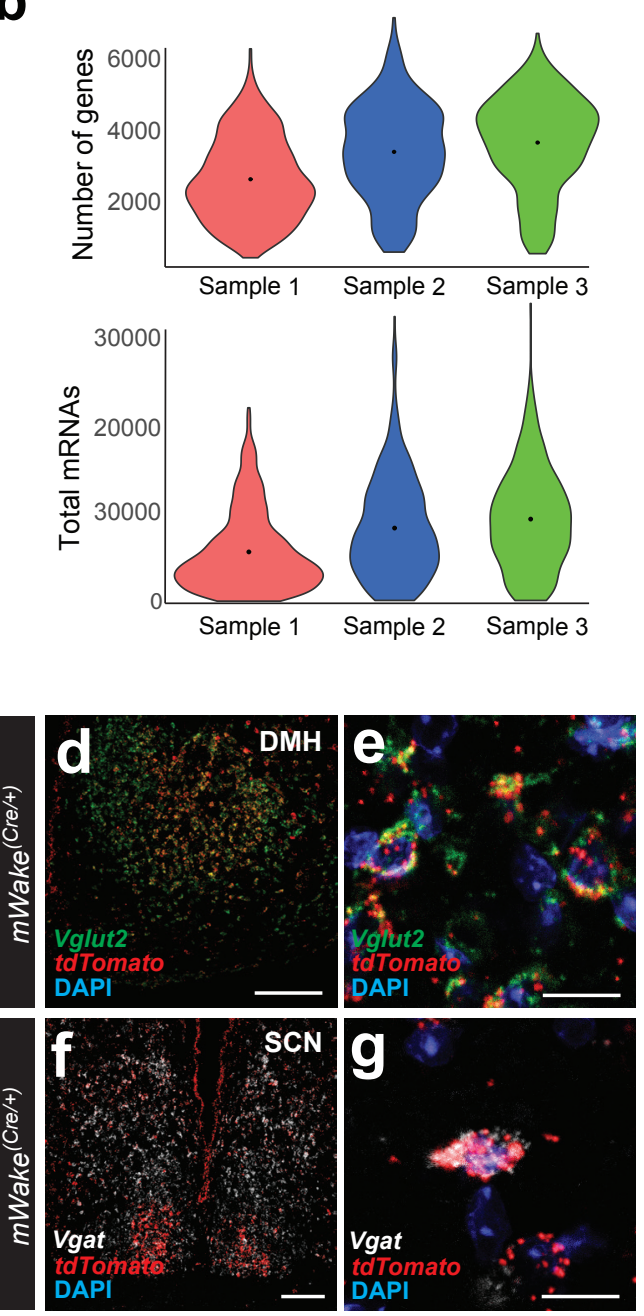
bioRxiv preprint doi: https://doi.org/10.1101/2020.03.12.989921; this version posted May 30, 2020. The copyright holder for this preprint (which was not certified by peer review) is the author/funder. All rights reserved. No reuse allowed without permission.
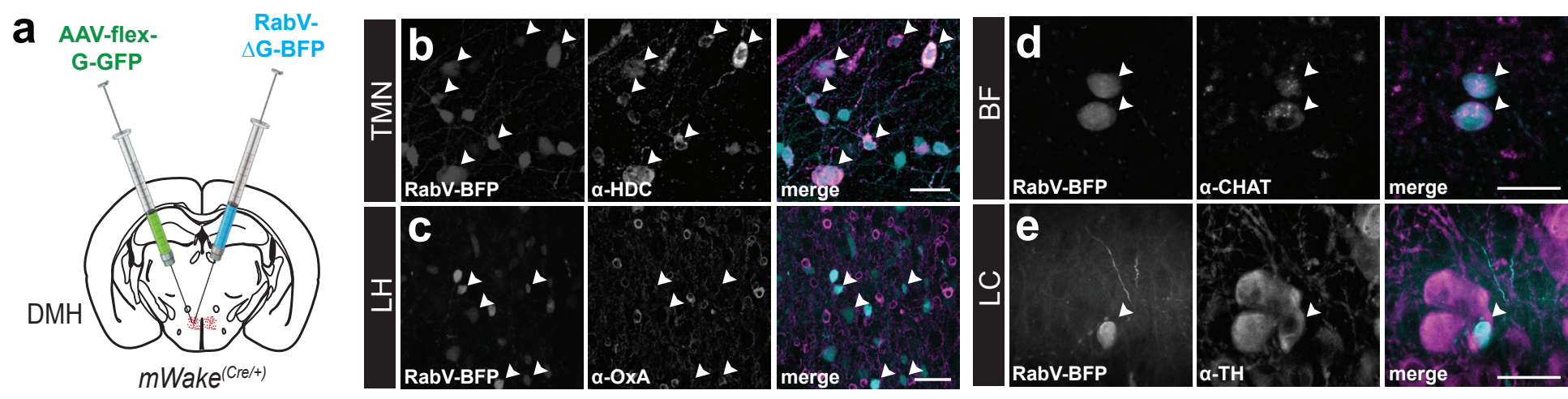

f

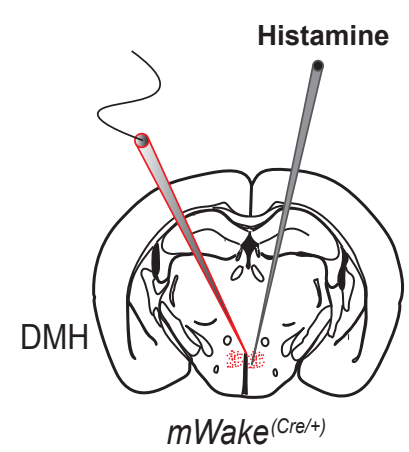

i

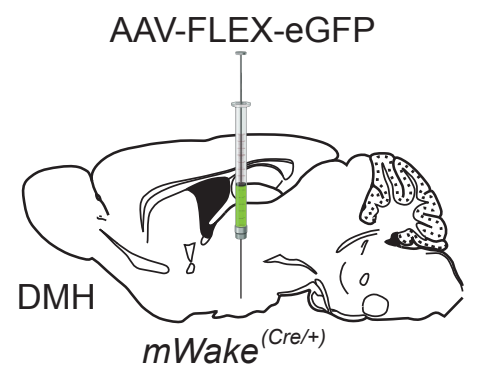

g

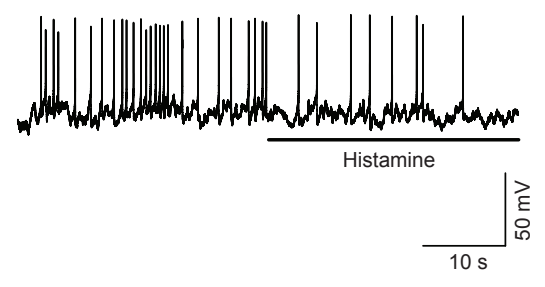

h
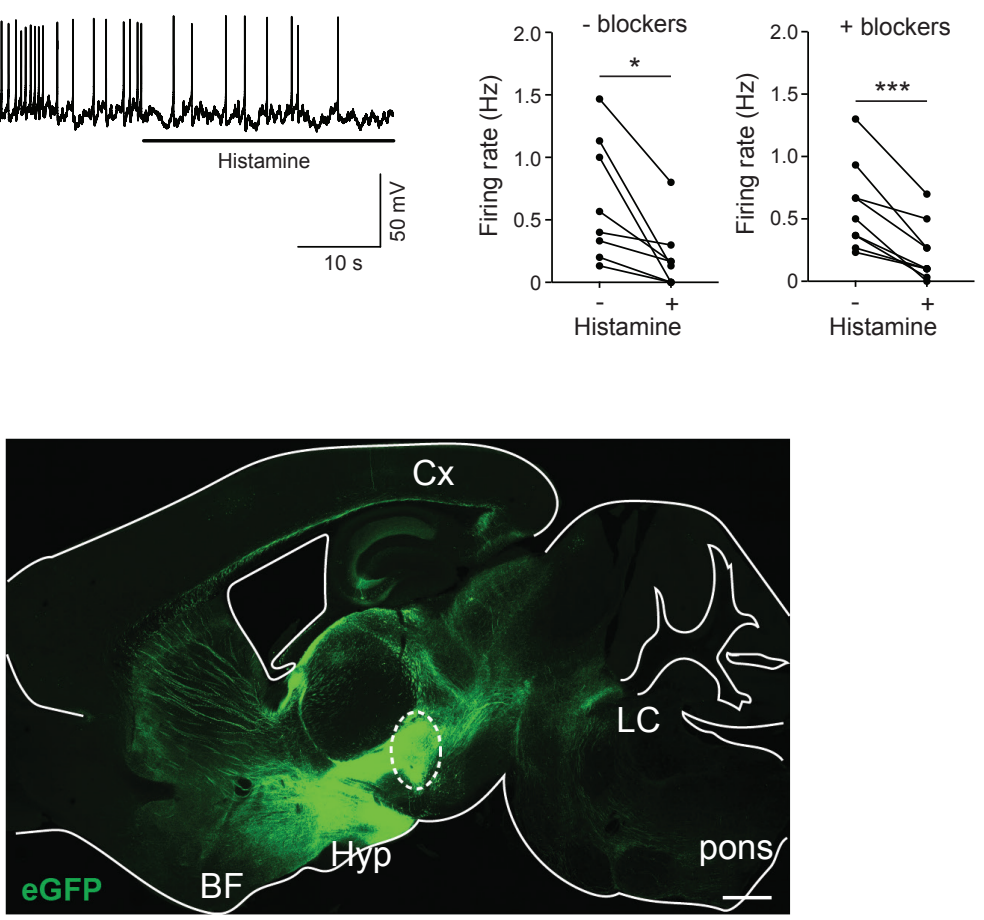
bioRxiv preprint doi: https://doi.org/10.1101/2020.03.12.989921; this version posted May 30, 2020. The copyright holder for this preprint (which was not certified by peer review) is the author/funder. All rights reserved. No reuse allowed without permission.

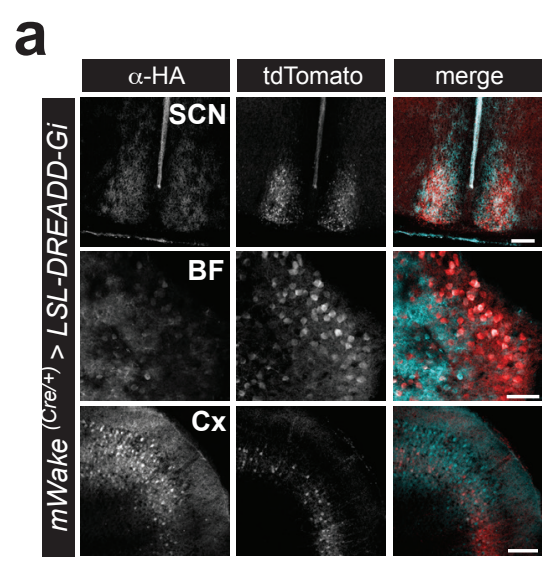

b

C
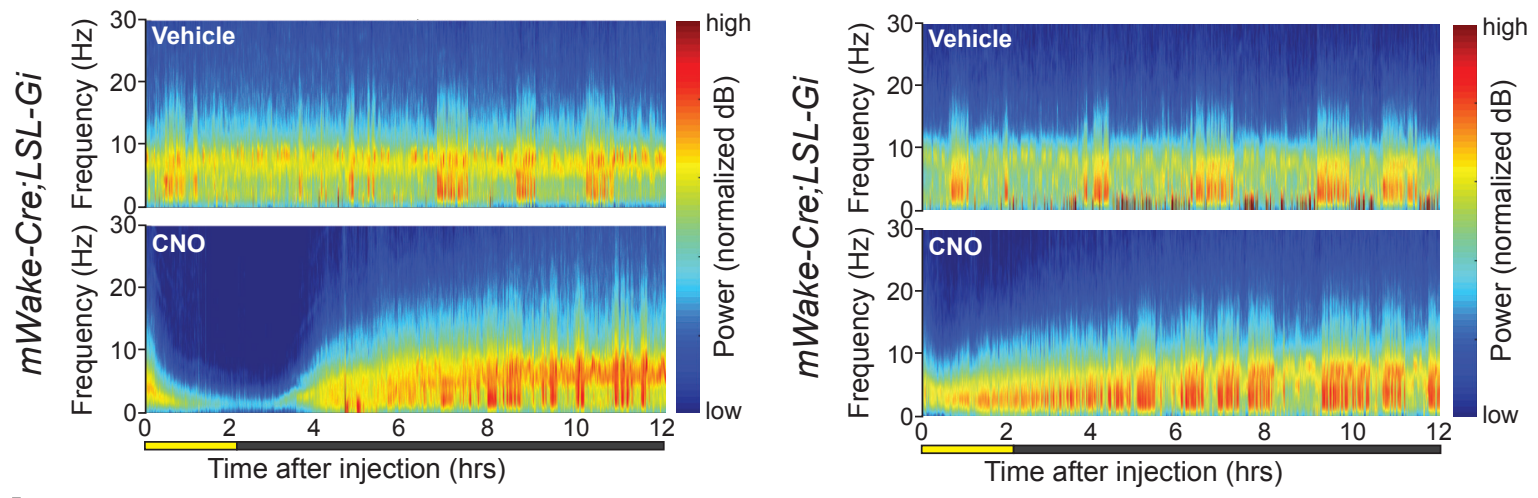

d

e
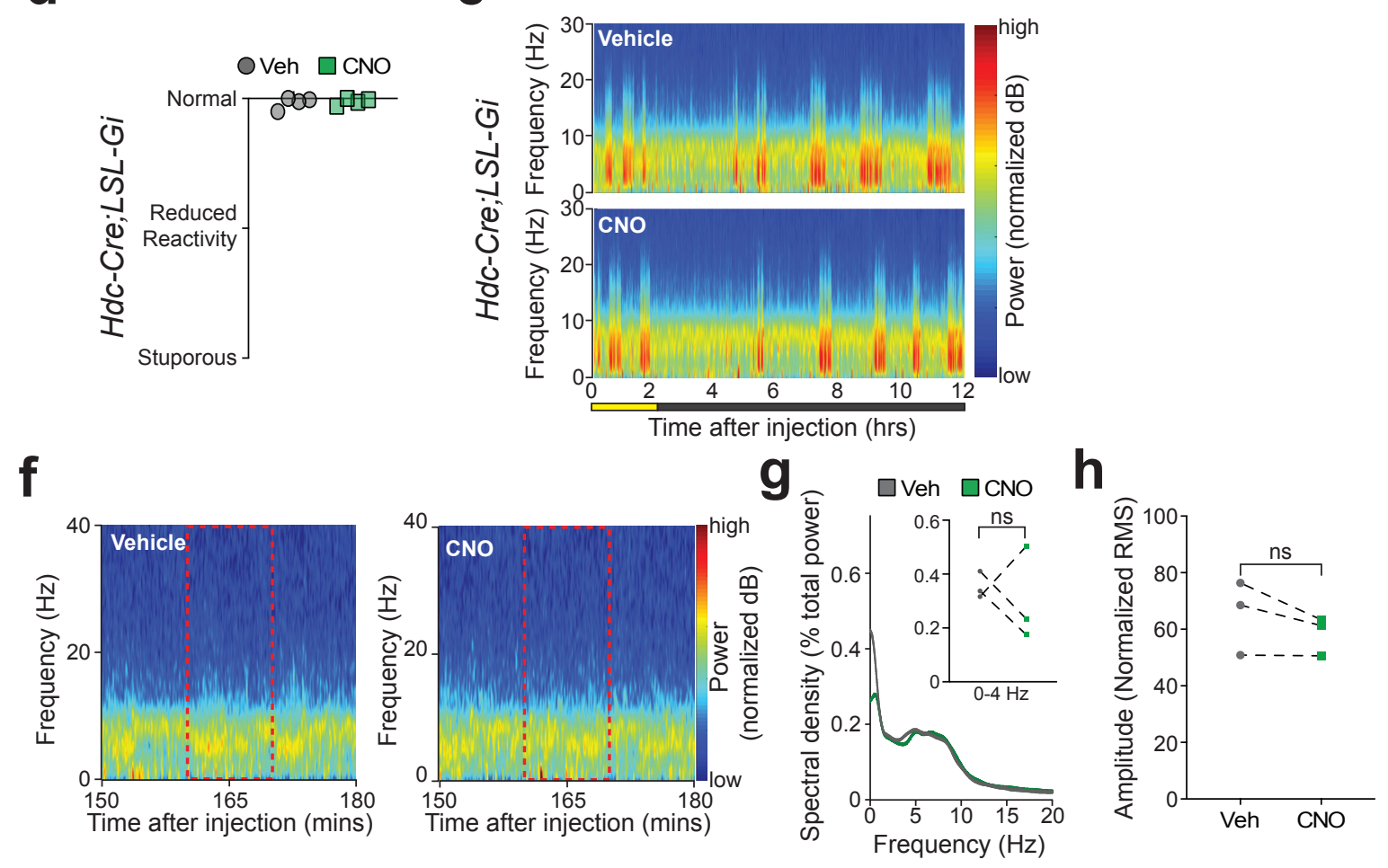